\title{
Advances in in vitro folliculogenesis in domestic ruminants
}

\author{
José Ricardo de Figueiredo ${ }^{1, \S}$, Jesús Cadenas ${ }^{1}$, Laritza Ferreira de Lima ${ }^{1}$, Regiane Rodrigues Santos $^{2}$ \\ ${ }^{1}$ Laboratory of Manipulation of Oocytes and Preantral Follicles, Faculty of Veterinary, State University of Ceara, Fortaleza CE, \\ Brazil. \\ ${ }^{2}$ Schothorst Feed Research, Lelystad, The Netherlands.
}

\begin{abstract}
The in vitro follicle culture (IVFC) represents an outstanding tool to enhance our understanding of the control of folliculogenesis and to allow the future use of a large number of immature oocytes enclosed in preantral follicles (PFs) in assisted reproductive techniques in humans as well as in others mammalian species including the ruminants. So far, the best results of IVFC were reported from mice with the production of live offspring from primordial follicles cultured in vitro. Live birth has been obtained after the in vitro culture of bovine early antral follicles. However, in other ruminant species, these results have been limited to the production of a variable number of mature oocytes and low percentages of embryos after in vitro culture of goat, buffalo and sheep isolated secondary preantral follicles. The present review presents and discusses the main findings, limitations, and prospects of in vitro folliculogenesis in ruminants focusing on bovine, caprine, and ovine species.
\end{abstract}

Keywords: folliculogenesis, preantral follicle, ruminant, in vitro development.

\section{Introduction}

Ruminants are distributed worldwide and have social and economic importance for many countries, contributing to circa $76 \%$ of the global livestock biomass (FAO, 2010). To keep animal production within expected levels, nutrition and reproduction are the key factors. Regarding reproduction, it is essential to understand the mechanisms involved in the formation of the gametes, as well as their development to improve techniques and outcomes (Thatcher, 2017).

At birth, ruminant ovaries contain thousands of immature oocytes, being the vast majority of them enclosed in preantral follicles, that represent the main ovarian oocyte reserve. Despite this large follicle population, most of them (approximately $99.9 \%$ of the follicles) will undergo atresia during folliculogenesis. The regulation of folliculogenesis in the preantral follicle phase is an extremely complex process and involves the interaction among endocrine, paracrine and autocrine factors, as previously revised (Figueiredo et al., 2011, 2018).

The Assisted Reproductive Techniques (ARTs) are of great importance for both basic and applied research. Basic, or fundamental, research is crucial to understand the physiology of reproduction (Smith et al., 2014). Applied research helps to overcome severe infertility either in male or female, as well as to increase the genetic selection rate of highly producing animals (Tan et al., 2017). Among the ARTs that aim to optimize the use in the future of the large ovarian oocyte reserve it is important to highlight the in vitro follicle culture (IVFC) (Green and Shikanov, 2016; Figueiredo et al., 2018). This technique represents an outstanding tool to enhance our understanding of the control of folliculogenesis and to allow the future use of a large number of immature oocytes enclosed in PFs in ARTs in humans as well as in others mammalian species. Interestingly, the production of live offspring from primordial follicles cultured in vitro has been successfully achieved in mice, and it was first reported in 1989 (Eppig and Schroeder, 1989). However, in ruminants, the results have been limited to the production a low percentage of embryos after in vitro culture of goat, buffalo, and sheep secondary preantral follicles (Silva et al., 2016). Therefore, this review presents and discusses the main findings, limitations, and prospects of in vitro folliculogenesis in ruminants focusing on bovine, caprine, and ovine species.

\section{Overview of follicle structure and populations and folliculogenesis regulation in vivo}

It is well known that mammalian ovaries contain from thousands to millions of follicles whereby approximately ninety percent of this population is represented by preantral follicles usually classified as primordial, intermediate, primary and secondary follicles. Despite this large follicle population, the vast majority of them will be eliminated by a physiological process called atresia during folliculogenesis. Folliculogenesis is the physiological process of activation, growth, and maturation of the ovarian follicle. The regulation of folliculogenesis involves a complex interaction among endocrine, paracrine and autocrine factors which in turn affects steroidogenesis, angiogenesis, basement membrane turnover, follicular atresia, oocyte growth, and maturation as well as the proliferation and differentiation of follicular cells (Figueiredo et al., 2018). In the ovary, the distribution of the regulating factors (ligands and their corresponding receptors) varies among follicular compartments (oocyte, granulosa, and theca cells) and significant changes in gene expression pattern among follicular categories (Yoon et al., 2006) have been reported. The control of folliculogenesis is extremely complex because the regulating factors act by binding to different types of receptors that activate distinct signaling pathways and, sometimes different ligands share the same receptors (Figueiredo et al., 2018). 
Also, there are complex interactions among cell signaling pathways which eventually control gene expression that determines cell survival or death, quiescence or proliferation. Therefore, follicular maturation or atresia will depend on a delicate balance between stimulatory and inhibitory stimuli. Folliculogenesis during the preantral follicle phase can be divided into three steps: (i) the activation (recruitment) of primordial follicles, i.e., transition from primordial (quiescent follicle) to growing follicles (intermediate and primary follicle); (ii) development of primary and secondary follicles; (iii) transition from preantral to antral follicle. In general, it has been stated that the growth of primordial follicles up to the early antral stage is pituitary independent, being probably controlled by autocrine/paracrine mechanisms and modulated by gonadotrophins (for review see Figueiredo et al. 2018). With the understanding of the factors involved in the early folliculogenesis, it will be possible to optimize the use of the large oocyte ovarian reserve in ARTs in humans as well as in other mammalian species. Among these technologies, it is important to highlight the IVFC, as discussed in the coming sections.

\section{In vitro follicle culture (IVFC)}

\section{Purpose, applications, and type of culture systems}

Taking into account that the vast majority of follicles will be eliminated by atresia in case they remain in the ovary the ultimate goal of IVFC is to rescue preantral follicles from the ovary before they become atretic, and culture them up to maturational stages for further in vitro fertilization and embryo production, functioning as an artificial ovary. This technology has some current and future applications such as: (i) to study the control of early folliculogenesis; (ii) to complement other reproductive technologies (e.g., in vitro embryo production, nuclear transfer, etc); (iii) to create gamete banks from endangered species and breeds; (iv) to preserve the fertility in individuals subjected to cancer treatment; infertility treatment (human), and (v) to aid in studies on reproductive toxicology (Figueiredo et al., 2011).

Basically, there are two ways to culture preantral follicles: in the isolated form or enclosed in ovarian tissue. Isolated follicles can be cultured in a two-dimensional system which means the follicle is placed on the surface, such as plastic or on an extracellular matrix for instance collagen gel or follicles can be cultured in a 3D system enclosed in an extracellular matrix such as alginate. In situ cultures, on the other hand, have been performed using ovarian fragments or the whole ovary (Figueiredo et al., 2011).

\section{Main endpoints used to evaluate the efficiency of IVFC}

The efficiency of IVFC can be evaluated using the following endpoints that are crucial for understanding folliculogenesis regulation: follicular survival (morphology/viability); follicular activation and progression through folliculogenesis; oocyte and follicular growth; hormone production; gene expression for key factors (Ligands/receptors); antrum formation; production of fully grown $(>110 \mu \mathrm{m})$ meiotically competent oocytes; finally, it is also possible to evaluate the oocyte developmental competence through the production of embryos and live offspring (Fig. 1).

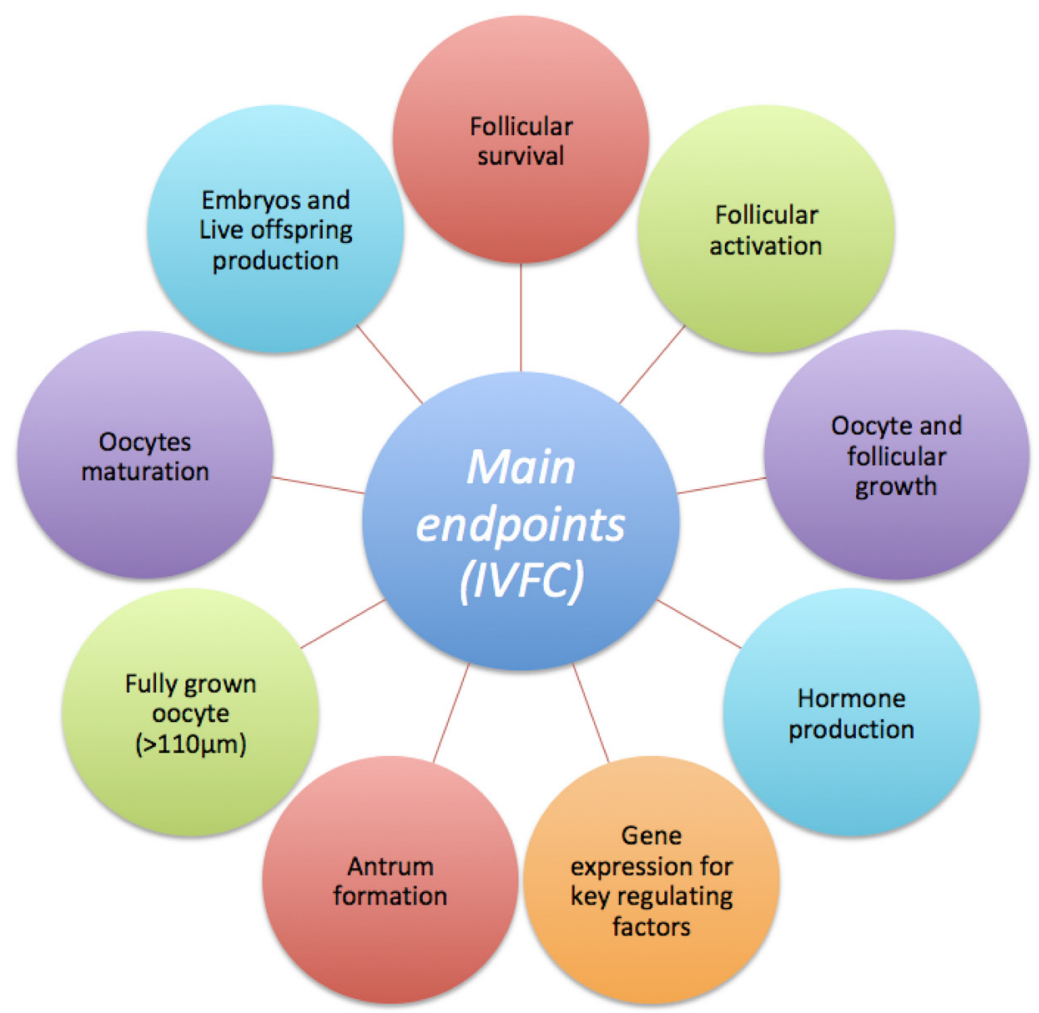

Figure 1. The main endpoints used to evaluate the efficiency of IVFC in mammals. 


\section{Main progresses in the in vitro culture of ruminant follicles}

\section{Bovine}

Although live birth has been produced after the in vitro culture of cumulus-granulosa cell complexes from early antral follicles $(0.3-0.7 \mathrm{~mm}$ in diameter $)$ (Hirao et al., 2004; Yamamoto et al., 1999), the bovine species is the one facing more difficulties when it comes to move forward in the field of IVFC. The best achievement so far from PFs is the antrum formation after the in vitro culture of primordial and intermediate follicles $(<40 \mu \mathrm{m}$ diameter) enclosed in ovarian tissue into secondary follicles $(\sim 110 \mu \mathrm{m}$ in diameter $)$, followed by the isolation and in vitro culture of those in vitro grown secondary follicles (McLaughlin and Telfer, 2010). Araújo et al. (2014b) made a profound review on this subject, however, significant advances have been published in recent years that are worth mentioning. The main results are summarized in Table 1.

Early PFs, i.e., primordial and primary follicles are usually cultured in vitro enclosed in ovarian tissue (in situ). With this system, Jorssen et al. (2014) showed that neutral red (NR) staining can be used to facilitate follicle evaluation during a short-term culture (6 days) without affecting follicle developmental competence regardless the oxygen tension (high: $20 \% \mathrm{O} 2$; low: $5 \%$ $\mathrm{O} 2)$. Regarding culture media supplements, the addition of either growth and differentiation factor 9 (GDF-9) or basic fibroblast growth factor (bFGF) to a medium containing follicle stimulating hormone (FSH) enhanced the beneficial effect of FSH alone in terms of follicle morphology, activation and growth (follicle diameter from $\sim 25 \mu \mathrm{m}$ on D0 to $\sim 90 \mu \mathrm{m}$ on D22 of culture) (Tang et al., 2012).

In addition, the base medium itself can affect follicle viability and development. In fact, Jimenez et al. (2016) stated that $\alpha \mathrm{MEM}$ is more effective maintaining follicle viability and promoting follicle growth than both TCM199 and McCoy media. Conversely, Castro et al. (2014) recommended the use of TCM199 and McCoy media for the culture of fresh and vitrified bovine ovarian tissue, respectively, meaning that the process to which the ovarian tissue is submitted prior culture must also be considered before selecting a base culture medium.

Besides growth factors and hormones, the role of cytokines during folliculogenesis, such as tumor necrosis factor-alpha (TNF- $\alpha$ ) and interleukin-1 (IL-1), as well as their distribution within the bovine ovary has been investigated. Thereby, proteins of the TNF- $\alpha$ system members, i.e., TNF- $\alpha$ and its receptors (TNFR1/TNFR2), have been detected in oocytes from all follicular categories, in granulosa cells from the secondary stage onwards, and in theca cells at the antral stage. Nonetheless, the addition of TNF- $\alpha$ has shown to reduce follicle survival after 6 days of culture (Silva et al., 2017a). Moreover, proteins of the IL-1 system, i.e., IL-1 $\beta$ and its receptors (IL-1RI, IL-1RII, and IL-1RA) have been detected in oocytes and granulosa cells from all follicular categories and in theca cells at the antral stage. But unlike TNF- $\alpha$, the addition of IL- $1 \beta$ favored follicular activation and development after 6 days of culture (Passos et al., 2016). The in situ system has also complemented other biotechnologies such as ovarian tissue xenotransplantation. As a matter of fact, a $24 \mathrm{~h}$ culture of bovine ovarian tissue in the presence of VEGF prior to xenotransplantation into mice enhanced follicle survival for up to 2 weeks (Langbeen et al., 2016).

As seen in the in situ system, bovine follicles cultured in vitro in the isolated form are also affected by the base medium composition. Rossetto et al. (2012) obtained greater follicle growth, viability and antrum formation (60\%), when using TCM199 medium compared to both $\alpha-M E M$ and McCoy media with the same supplementations and medium replacement regime i.e., half of the medium $(75 \mu \mathrm{l})$ was refreshed every 4 days. However, when another medium replacement regime was used (addition of $5 \mu \mathrm{l}$ of fresh culture medium to an initial volume of $50 \mu$ l every other day), $\alpha$-MEM became equivalent to TCM199 in terms of follicular growth and antrum formation (Araújo et al., 2015).

Furthermore, different supplements have shown to exert a positive effect on follicular growth and/or antrum formation of isolated secondary follicles when added alone, such as: FSH (Passos et al., 2013; Silva et al., 2014), bone morphogenetic protein-15 (BMP-15) (Passos et al., 2013), VEGF (Araújo et al., 2014a), insulin (Rossetto et al., 2016), and alpha lipoic acid (ALA) (Zoheir et al., 2017). Nevertheless, the combination of FSH and BMP-15 or Activin A have not improved the IVFC outcome (Passos et al., 2013; Silva et al., 2014, respectively)

Less developed follicular categories, i.e., primordial and primary follicles have successfully reached the antral stage in vitro after 21 days of culture: primordial follicles $(<40 \mu \mathrm{m})$ cultured in a two-step system consisting on 6 days of in situ culture followed by 15 days of isolated 2D culture (Act $\mathrm{A}$ was added to isolated follicles) (McLaughlin and Telfer, 2010); and primary follicles $(50-70 \mu \mathrm{m})$ in an isolated $3 \mathrm{D}$ system (collagen matrix) in the presence of FSH, luteinizing hormone (LH), estradiol (E2), epidermal growth factor (EGF) and bFGF (Sun and Li, 2013).

In summary, bovine PFs have been able to remain viable during in vitro culture and to form antrum from the primordial stage. Nevertheless, no oocyte meiotic maturation has been accomplished yet. Therefore, future research should focus on the specific factors involved on the in vitro obtention of metaphase II oocytes in this species. Furthermore, besides the fact that bovine is indicated as model for reproductive toxicology studies, especially during oocyte maturation (Santos et al., 2014), not so much has been done with PFs.

Ovine

Among the three main species of domestic ruminants (bovine, ovine and caprine), the ovine species is the one where the IVFC has improved the most in the last years (Table 2), probably because is the most used animal model for humans. Hence, it has been reported the production of a relatively high rate of metaphase II (MII) 
oocytes (Arunakumari et al., 2010; Barboni et al., 2011) and a low number of embryos at the morula stage after in vitro fertilization (IVF) or parthenogenetic activation of in vitro cultured isolated secondary follicles (Arunakumari et al., 2010; Barboni et al., 2011; Luz et al., 2013). In this sense, Arunakumari et al. (2010) obtained $68 \%$ of MII oocytes from which $25 \%$ developed to the 2-cell embryo stage, and $16 \%$ reached the morula stage (out of the cleaved embryos) after the IVF of oocytes derived from the isolated PFs (200-400 $\mu \mathrm{m})$ cultured in medium TCM 199 containing thyroxin (T4), FSH, IGF-I, and GH for 6 days. Barboni et al. (2011) also produced competent oocytes from smaller PFs (170 $\mu \mathrm{m})$ cultured for 12 days in $\alpha$-MEM with fetal calf serum (FCS) and FSH, although after IVF only $10 \%$ of the resulting embryos reached $>16$-cell stage. Interestingly, in vitro grown oocytes from $360 \mu \mathrm{m}$ early antral follicles (AFs) (final follicle diameter at the end of the culture) presented similar methylation pattern and developmental capability than their in vivo grown counterparts (early AFs with the same diameter). However, oocytes from both in vivo and in vitro grown early AFs $(360 \mu \mathrm{m})$ presented low competence compared to oocytes from in vivo grown AFs (6 mm) (Barboni et al., 2011).

Aiming to optimize the current IVFC systems and to understand better the process of folliculogenesis in vitro, several substances and/or culture systems have been tested mainly on isolated secondary follicles $(>200$ $\mu \mathrm{m})$. Thus, it has been shown that rutin alone could potentially replace the combination of the three commonly used antioxidants in culture medium (transferrin, selenium and ascorbic acid), and consequently simplify its composition (Lins et al., 2017). Also, leukemia inhibitory factor (LIF) promoted the rupture of the basement membrane without affecting oocyte maturation or embryo development since 8-cell parthenotes were produced (Luz et al., 2012). The association of LIF and kit ligand (KL), on the other hand, stimulated oocyte meiotic resumption and even a morula was produced after IVF. Nevertheless, this association did not improve the results obtained by LIF alone in terms of oocyte maturation and embryo production (Luz et al., 2013). Likewise, the addition of human leptin to the culture medium increased follicular daily growth but did not affect oocyte maturation (Kamalamma et al., 2016).

Despite all these advances on IVFC of secondary follicles, oocyte maturation and embryo production rates are still far below the results obtained from follicles entirely grown in vivo. On this regard, several studies with this follicular category have shown that IVFC (isolated follicles in a 2D system) negatively affect the expression pattern and/or level of genes related to oocyte survival and development such as P450 aromatase (Lakshminarayana et al., 2014); B-cell leukemia/lymphoma $-2(\mathrm{Bcl} 2)$ and $\mathrm{Bcl} 2$-associated X protein (Bax) (Praveen Chakravarthi et al., 2015); connexins 32 and 43 (CX32 and CX43) (Chakravarthi et al., 2016a); cyclin B1 (CCNB1) and cyclin D1 (CND1) (Chakravarthi et al., 2016b); and GDF-9 and BMP-15 (Kona et al., 2016).

Early PFs (primordial and primary follicles) are usually cultured in situ (Bertoldo et al., 2014), although they have been cultured in the isolated form as well within a 3D matrix (alginate) (Sadeghnia et al., 2016). These follicular categories have provided some knowledge about what factors mediate follicle activation. Nowadays, increasing evidence suggests that some of them pertain to transforming growth factor $\beta$ (TGF- $\beta$ ) superfamily (Knight and Glister, 2006), which includes the bone morphogenetic proteins (BMPs). Nonetheless, the addition of BMP4 during the culture of ovarian cortex pieces did not affect follicle activation but enhanced follicle survival and growth (Bertoldo et al., 2014). Also, the stiffness of the environment surrounding the follicles seems to affect their activation and further development. The encapsulation of ovarian cortex in 0.5 or $1 \%$ alginate was detrimental for follicle development, while the encapsulation of isolated primordial follicles in $2 \%$ alginate potentiated their growth (Sadeghnia et al., 2016).

As it was introduced above, one of the multiple potential applications of IVFC is to serve as an in vitro model for toxicology assays. It is noteworthy that in this species, this technique has been used already for this purpose. Thus, it was determined the minimum concentration of some metabolic stressors that impaired preantral follicle function: $300 \mu \mathrm{M}$ ammonia, $8 \mathrm{mM}$ urea, $210 \mu \mathrm{M}$ non-esterified fatty acids (NEFA) $(30 \mu \mathrm{M}$ stearic acid $+60 \mu \mathrm{M}$ palmitic acid $+120 \mu \mathrm{M}$ oleic acid), and $0.75 \mu \mathrm{M} \beta$-hydroxybutyric acid (BHB) (Nandi et al., 2017). Moreover, it was shown that aqueous extracts of the plant Justicia insularis were able to maintain follicle morphology and to stimulate primordial follicle activation during the in vitro culture of PFs enclosed in ovarian tissue for 7 days (Mbemya et al., 2017). Early PFs (primordial and primary follicles) are usually cultured in situ (Bertoldo et al., 2014), although they have been cultured in the isolated form as well within a 3D matrix (alginate) (Sadeghnia et al., 2016). These follicular categories have provided some knowledge about what factors mediate follicle activation. Nowadays, increasing evidence suggests that some of them pertain to transforming growth factor $\beta$ (TGF- $\beta$ ) superfamily (Knight and Glister, 2006), which include the bone morphogenetic proteins (BMPs). Nonetheless, the addition of BMP4 during the culture of ovarian cortex pieces did not affect follicle activation but enhanced follicle survival and growth (Bertoldo et al., 2014). Also, the stiffness of the environment surrounding the follicles seems to affect their activation and further development. The encapsulation of ovarian cortex in 0.5 or $1 \%$ alginate was detrimental for follicle development, while the encapsulation of isolated primordial follicles in $2 \%$ alginate potentiated their growth (Sadeghnia et al., 2016).

In general, in spite of the progress made on this topic for the last few years, the developmental competence of the oocytes obtained from in vitro cultured PFs is still low relative to their in vivo counterparts. Even though a considerable proportion of oocytes from PFs are nowadays able to grow and reach the MII stage in vitro, the fact that embryos are capable of developing only until morulae highlights the need for further investigation regarding oocyte cytoplasmic maturation. 
Table 1. Chronological advances in in vitro culture of bovine preantral follicles*.

\section{References/Base medium}

\section{Follicle Category/Culture system}

McLaughlin and Telfer, 2010 - McCoy's $5 \mathrm{a}$ Bicarbonate (20mM Hepes, 3mM GLUT, $0.1 \%$ BSA, $2.5 \mu \mathrm{g} / \mathrm{ml}$ TRAN, $4 \mathrm{ng} / \mathrm{ml} \mathrm{SEL}, 10 \mathrm{ng} / \mathrm{ml}$ INS and $50 \mu \mathrm{g} / \mathrm{ml}$ $\mathrm{AA})$

Tang et al., 2012 - $\alpha$-MEM (100 ng/ml FSH, $1.25 \mathrm{mg} / \mathrm{ml} \mathrm{BSA,} 1 \mu \mathrm{g} / \mathrm{ml}$ INS, 5.5 $\mu \mathrm{g} / \mathrm{ml}$ TRAN and $5 \mathrm{ng} / \mathrm{ml}$ SEL)

Rossetto et al., 2012 - Base media (20mM Hepes, 3mM GLUT, 0.1\% BSA, 2.5 $\mu \mathrm{g} / \mathrm{ml}$ TRAN, $4 \mathrm{ng} / \mathrm{ml} \mathrm{SEL}, 10 \mathrm{ng} / \mathrm{ml} \mathrm{INS}, 50 \mu \mathrm{g} / \mathrm{ml} \mathrm{AA}, 100 \mathrm{ng} / \mathrm{ml}$ Act A and $100 \mathrm{ng} / \mathrm{ml} \mathrm{FSH})$

Passos et al., 2013 - $\alpha$-MEM $(10 \mu \mathrm{g} / \mathrm{ml} \mathrm{INS,} 5.5 \mu \mathrm{g} / \mathrm{ml}$ TRAN, $5 \mathrm{ng} / \mathrm{ml} \mathrm{SEL}, 3$ $\mathrm{mg} / \mathrm{ml} \mathrm{BSA}, 2 \mathrm{mM}$ GLUT, $2 \mathrm{mM}$ HYP, $50 \mu \mathrm{g} / \mathrm{ml} \mathrm{AA}$ )

Sun and Li, 2013 - $\alpha$-MEM (0.23 mM PYR, 1.5 mM GLUT, 2 mM HYP, 7.5\% FBS, $5 \mu \mathrm{g} / \mathrm{ml} \mathrm{INS,} 5 \mu \mathrm{g} / \mathrm{ml}$ TRAN, $5 \mathrm{ng} / \mathrm{ml} \mathrm{SEL})$

Silva et al., 2014 - $\alpha$-MEM ( $3 \mathrm{mg} / \mathrm{mL}$ BSA, $10 \mu \mathrm{g} / \mathrm{ml} \mathrm{INS,} 5.5 \mu \mathrm{g} / \mathrm{ml}$ TRAN, 5 $\mathrm{ng} / \mathrm{ml} \mathrm{SEL}, 2 \mathrm{mM}$ GLUT, $2 \mathrm{mM}$ HYP, $50 \mu \mathrm{g} / \mathrm{ml} \mathrm{AA}$ )

Araújo et al., 2014a - $\alpha$-MEM ( $3 \mathrm{mg} / \mathrm{mL}$ BSA, $10 \mu \mathrm{g} / \mathrm{ml} \mathrm{INS}, 5.5 \mu \mathrm{g} / \mathrm{ml}$ TRAN, $6.7 \mathrm{ng} / \mathrm{ml} \mathrm{SEL}, 2 \mathrm{mM}$ GLUT, $2 \mathrm{mM}$ HYP, $50 \mu \mathrm{g} / \mathrm{ml}$ AA, $100 \mathrm{ng} / \mathrm{ml}$ FSH)

Primordial - In situ (6 days) Secondary Isolated $2 \mathrm{D}$ (15 days)

Preantral - In situ (22 days)

Secondary - Isolated 2D (16 days)

Secondary - Isolated 2D (12 days)

Primary - Isolated 3D (Type I collagen - 21 days

Secondary - Isolated 2D (18 days)

Secondary - Isolated 2D/3D (alginate - 32 days)

Jorssen et al., 2014 - McCoy's 5a (10mM Hepes, 3mM GLUT; 0.1\% BSA, 5 $\mu \mathrm{g} / \mathrm{ml}$ TRAN, $5 \mathrm{ng} / \mathrm{ml} \mathrm{SEL}, 5 \mu \mathrm{g} / \mathrm{ml}$ INS, $50 \mu \mathrm{g} / \mathrm{ml}$ AA)

Castro et al., 2014 - Base media (3mM GLUT; 2mM HYP, 0.1\% BSA, $2.5 \mu \mathrm{g} / \mathrm{m}$ TRAN, $4 \mathrm{ng} / \mathrm{ml} \mathrm{SEL}, 10 \mathrm{ng} / \mathrm{ml}$ INS, $50 \mu \mathrm{g} / \mathrm{ml}$ AA)

Araújo et al., 2015 - $\alpha$-MEM or TCM199 $(3 \mathrm{mg} / \mathrm{mL}$ BSA, $10 \mu \mathrm{g} / \mathrm{ml} \mathrm{INS}, 5.5$ $\mu \mathrm{g} / \mathrm{ml}$ TRAN, $5 \mathrm{ng} / \mathrm{ml} \mathrm{SEL}, 2 \mathrm{mM}$ GLUT, $2 \mathrm{mM}$ HYP, $50 \mu \mathrm{g} / \mathrm{ml} \mathrm{AA}$ )

Rossetto et al., 2016 - TCM-199-HEPES (3mM GLUT, $0.1 \%$ BSA, $2.5 \mu \mathrm{g} / \mathrm{ml}$ TRAN, $4 \mathrm{ng} / \mathrm{ml}$ SEL, $10 \mathrm{ng} / \mathrm{ml}$ INS, $50 \mu \mathrm{g} / \mathrm{ml} \mathrm{AA}, 100 \mathrm{ng} / \mathrm{ml}$ Act A, $100 \mathrm{ng} / \mathrm{ml}$ FSH

Jimenez et al., 2016 - Base media (20 mM Hepes, 3mM GLUT, 0.1\% BSA, 2.5 $\mu \mathrm{g} / \mathrm{ml}$ TRAN, $4 \mathrm{ng} / \mathrm{ml}$ SEL, $10 \mathrm{ng} / \mathrm{ml} \mathrm{INS}, 50 \mu \mathrm{g} / \mathrm{ml} \mathrm{AA}$

Passos et al., 2016 - $\alpha$-MEM (2 mM GLUTA, 2 mM HYPO, 3 mg/ml BSA, 10 $\mu \mathrm{g} / \mathrm{ml}$ INS, $5.5 \mu \mathrm{g} / \mathrm{ml}$ TRANS, $5 \mathrm{ng} / \mathrm{ml}$ SEL)

Silva et al., 2017a - $\alpha$-MEM( 2 mM GLUTA, 2 mM HYPO, $1.25 \mathrm{mg} / \mathrm{ml}$ BSA, 10 $\mu \mathrm{g} / \mathrm{ml}$ INS, $5.5 \mu \mathrm{g} / \mathrm{ml}$ TRANS, $5 \mathrm{ng} / \mathrm{ml}$ SEL)

Zoheir et al., 2017 - TCM-199 (10\% Newborn Calf Serum, 0.23 mM PYRU, 1\% ITS, $2.2 \mathrm{~g} / 1$ sodium bicarbonate, $100 \mathrm{ng} / \mathrm{ml} \mathrm{FSH,} 100 \mathrm{ng} / \mathrm{ml}$ EGF

Preantrat $-\ln \operatorname{situ}(6 \mathrm{days})$

Preantral - In situ (5 days)

Secondary - Isolated 2D (32 days)

Secondary - Isolated 2D and 3D (Collagen - 18 days)

Preantral - In situ (7 days)

Preantral- In situ (6 days)

All follicular categories - In situ (6 days)

Secondary - Isolated 2D (15 days)

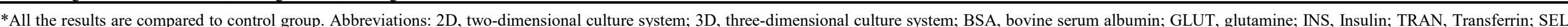

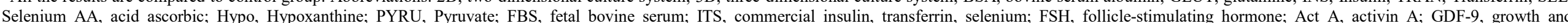

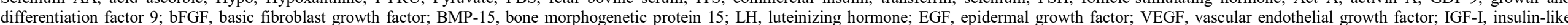
growth factor; GH, growth hormone; IL-1 $\beta$, interleukin-1 $\beta$; TNF- $\alpha$, tumor necrosis factor-alpha; ALA, alpha lipoic acid.
$100 \mathrm{ng} / \mathrm{ml}$ Act A or $50 \mathrm{ng} / \mathrm{ml}$ FSH - Primordial follicle Act A - $\uparrow$ follicle and oocyte growth and antrum formation

$200 \mathrm{ng} / \mathrm{ml}$ GDF-9 or $100 \mathrm{ng} / \mathrm{ml}$ bFGF - GDF-9+FSH and bFGF+FSH - $\downarrow$ apoptosis and $\uparrow$ activation; GDF9+FSH - $\uparrow$ follicle diameter

Different base media: aMEM, McCoy and TCM199 - TCM199 -

follicle viability, diameter and antrum formation

$50 \mathrm{ng} / \mathrm{ml} \mathrm{BMP-15}$ and $50 \mathrm{ng} / \mathrm{ml}$ FSH - BMP-15 and FSH alone -

follicular volume and antrum formation; BMP-15+FSH - $\uparrow$ atresia

$0.25 \mu \mathrm{g} / \mathrm{ml}$ FSH, $5 \mathrm{IU} / \mathrm{ml} \mathrm{LH,} 0.5 \mu \mathrm{g} / \mathrm{ml} \mathrm{E2,} 25 \mathrm{ng} / \mathrm{ml} \mathrm{EGF}$ and $50 \mathrm{ng} / \mathrm{ml} \mathrm{bFGF} \mathrm{-}$ All factors - $\uparrow$ follicle diameter and antrum formation

$100 \mathrm{ng} / \mathrm{ml}$ Act $\mathrm{A}$ and FSH $(50 \mathrm{ng} / \mathrm{ml}$ D0-D6; $100 \mathrm{ng} / \mathrm{ml}$ D7-D12; $200 \mathrm{ng} / \mathrm{ml}$ D13-D18) Sequential FSH - $\uparrow$ follicle growth; Act A and FSH alone $\uparrow$ follicle survival.

$100 \mathrm{ng} / \mathrm{ml} \mathrm{VEGF,} 50 \mathrm{ng} / \mathrm{ml} \mathrm{GH,} 50 \mathrm{ng} / \mathrm{ml} \mathrm{IGF-I} \mathrm{-} \mathrm{System} \mathrm{2D} \mathrm{X} \mathrm{3D} \mathrm{-}$

VEGF + 2D: $\uparrow$ diameter, antrum formation and growth rate, and maintained morphology

$50 \mu \mathrm{g} / \mathrm{ml}$ neutral red (NR) in the presence of: $5 \% \mathrm{O}_{2}$ vs. $20 \% \mathrm{O}_{2}$ - Follicle dynamics are not influenced by $\mathrm{O}_{2}$ tension

Different base media: McCoy, $\alpha$-MEM, and TCM199 (Fresh vs. Vitrified) TCM199 maintained morphology (Fresh tissue); McCoy $\uparrow$ growth and maintained viability (Vitrified tissue)

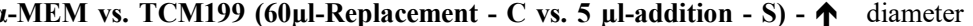
and antrum formation in TCM199-C vs $\alpha$-MEM-C.

$5,10 \mathrm{ng} / \mathrm{ml}$ or $\mu \mathrm{g} / \mathrm{ml}$ INS (2D) and $10 \mathrm{ng} / \mathrm{ml}$ INS+ FSH fixed $(100 \mathrm{ng} / \mathrm{ml}) \mathrm{vs}$. sequential (1 $\left.\mathbf{n g} / \mathrm{ml}_{\text {Do-D6 }} ; 10 \mathrm{ng} / \mathrm{ml}_{\text {D6-D12 }} ; 100 \mathrm{ng} / \mathrm{ml}_{\text {D12-D18) }}\right)-10 \mathrm{ng} / \mathrm{ml}$ INS alone or with fixed FSH $\uparrow$ diameter and daily growth

Different base media: aMEM, TCM19

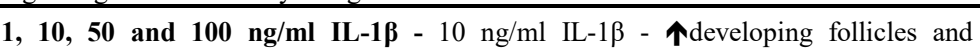
maintained normal morphology $\uparrow$ cell apoptosis and $10 \mathrm{ng} / \mathrm{ml}$ dexamethasone conserved follicle ultrastructure

100,250 or $500 \mu M$ ALA - ALA $\uparrow$ follicle growth and maintained viability
10,100 or $200 \mathrm{ng} / \mathrm{ml}$ TNF- $\alpha$ and $1,10,100$ or $200 \mathrm{ng} / \mathrm{ml} \mathrm{dexamethasone} \mathrm{-} \mathrm{TNF-} \alpha$ 
Table 2. Chronological advances in in vitro culture of ovine preantral follicles*.

\begin{tabular}{|c|c|c|}
\hline References/Base medium & $\begin{array}{l}\text { Follicle Category/Culture } \\
\text { system }\end{array}$ & Tested compounds/Main findings \\
\hline Arunakumari et al., 2010 - TCM199 Bicarbonate & $\begin{array}{l}\text { Secondary - Isolated 2D vs. 3D } \\
\text { (Agar-coated plates - } 6 \text { days) }\end{array}$ & $\begin{array}{l}\text { Different concentrations and association of ITS, IGF-I, insulin, GH, and TGF- } \beta \text { with } \mathbf{1} \boldsymbol{\mu g} / \mathbf{m l ~ T 4}+\mathbf{2} \boldsymbol{\mu g} / \mathbf{m l ~ F S H ~} \\
\text { (Microdrops vs. Agar gel) - Combination of } 1 \mu \mathrm{g} / \mathrm{ml} \mathrm{T4}, 2 \mu \mathrm{g} / \mathrm{ml} \mathrm{FSH}, 10 \mathrm{ng} / \mathrm{ml} \mathrm{IGF-I,} \mathrm{and} 1 \mathrm{mIU} / \mathrm{ml} \mathrm{GH} \boldsymbol{\uparrow} \text { antrum } \\
\text { formation, MII oocytes No differences between culture systems. } 25 \% 2-\text { cell embryo, and } 16 \% \text { morula. }\end{array}$ \\
\hline $\begin{array}{l}\text { Barboni et al., } 2011 \text { - } \alpha \text {-MEM (2\% FCS, 1\% ITS ,1 } \mu \mathrm{g} / \mathrm{ml} \\
\text { FSH) }\end{array}$ & $\begin{array}{l}\text { Secondary - Isolated 2D (14 } \\
\text { days) }\end{array}$ & $\begin{array}{l}\text { Oocyte nuclear epigenetic maturation from early antral follicles grown (in vivo vs. in vitro) - Similar methylation } \\
\text { profile in in vivo and in vitro. Similar oocyte maturation, fertilization, and embryo production from in vivo and in vitro } \\
\text { grown EAFs. } 32 \% \text { Fertilization, } 90 \%<16 \text {-cell embryos and } 10 \%>16 \text {-cell embryos }\end{array}$ \\
\hline $\begin{array}{l}\text { Luz et al., 2012 - } \alpha \text {-MEM(10 } \mu \mathrm{g} / \mathrm{ml} \text { INS, } 5.5 \mu \mathrm{g} / \mathrm{ml} \text { TRAN, } \\
5.5 \mathrm{ng} / \mathrm{ml} \text { SEL, } 3 \mathrm{mg} / \mathrm{ml} \text { BSA, } 2 \mathrm{mM} \text { GLUT, } 2 \mathrm{mM} \text { HYPO, } \\
50 \mu \mathrm{g} / \mathrm{ml} \mathrm{AA})\end{array}$ & $\begin{array}{l}\text { Secondary - Isolated 2D (18 } \\
\text { days) }\end{array}$ & $\begin{array}{l}10 \text { and } 50 \mathrm{ng} / \mathrm{ml} \text { LIF alone or in combination with FSH }\left(100 \mathrm{ng} / \mathrm{ml}_{\mathrm{D} 0-\mathrm{D} 6} ; 1000 \mathrm{ng} / \mathrm{ml}_{\mathrm{D} 6-\mathrm{D} 18} ; 200 \mathrm{ng} / \mathrm{ml}_{\mathrm{D} 13-\mathrm{D} 18)}\right)-50 \mathrm{ng} / \mathrm{ml} \\
\text { LIF } \text { 个extrusion. } 1 \text { Morula after IVF }\end{array}$ \\
\hline $\begin{array}{l}\text { Luz et al., 2013 - } \alpha \text {-MEM( }(10 \mu \mathrm{g} / \mathrm{ml} \text { INS, } 5.5 \mu \mathrm{g} / \mathrm{ml} \text { TRAN, } \\
5.5 \mathrm{ng} / \mathrm{ml} \mathrm{SEL}, 3 \mathrm{mg} / \mathrm{ml} \text { BSA, } 2 \mathrm{mM} \text { GLUT, } 2 \mathrm{mM} \text { HYPO, } \\
50 \mu \mathrm{g} / \mathrm{ml} \text { AA and } 50 \mathrm{ng} / \mathrm{ml} \text { LIF })\end{array}$ & $\begin{array}{l}\text { Secondary - Isolated 2D (18 } \\
\text { days) }\end{array}$ & $\begin{array}{l}50 \mathbf{~ n g} / \mathrm{ml} \text { IGF-I, and } 50 \mathrm{ng} / \mathrm{ml} \mathrm{KL} \text {, alone or in combination - KL } \uparrow \text { oocyte meiotic resumption. } 58.3 \% \text { eight-cell stage } \\
\text { parthenotes. }\end{array}$ \\
\hline $\begin{array}{l}\text { Bertoldo et al., 2014 - Waymouth MB } 752 / 1(25 \mathrm{mg} / \mathrm{l} \\
\text { PYR, } 6.25 \mu \mathrm{g} / \mathrm{ml} \mathrm{INS}, 6.25 \mu \mathrm{g} / \mathrm{ml} \text { TRAN, } 6.25 \mu \mathrm{g} / \mathrm{ml} \mathrm{SEL} \text {, } \\
1.25 \mathrm{mg} / \mathrm{ml} \mathrm{BSA}, 5.35 \mu \mathrm{g} / \mathrm{ml} \mathrm{LA})\end{array}$ & $\begin{array}{l}\text { Primordial and primary } \\
\text { follicles - In situ }(6 \text { days) }\end{array}$ & $\begin{array}{l}25,50 \text { or } 100 \mathrm{ng} / \mathrm{ml} \mathrm{BMP4} \text { with or without } 50 \mathrm{ng} / \mathrm{ml} \mathrm{FSH}-50 \mathrm{ng} / \mathrm{ml} \text { BMP4 - } \uparrow \text { follicle and oocyte diameters. Protected } \\
\text { primordial follicles from apoptosis }\end{array}$ \\
\hline $\begin{array}{l}-\mathrm{TCM} 199(1 \mu \mathrm{g} / \mathrm{ml} \mathrm{T} 4,2.5 \\
\mathrm{mIU} / \mathrm{ml} \mathrm{GH})\end{array}$ & econdary - Isolated 2D (6 days) & $\begin{array}{l}\text { Expression of P450 aromatase gene in cumulus cells and oocytes at different follicular stages (in vivo vs. in vitro). In } \\
\text { vitro culture } \downarrow \text { P450 aromatase expression. }\end{array}$ \\
\hline $\begin{array}{l}(1 \mu \mathrm{g} / \mathrm{ml} \mathrm{T} 4,2.5 \\
\mathrm{GH})\end{array}$ & econdary - Isolated 2D (6 days) & $\begin{array}{l}\text { Quantitative expression of } B c l 2 \text { and } B a x \text { ge } \\
\text { vitro) - } B c l 2 \text { to } B a x \text { ratio in oocytes and cumul }\end{array}$ \\
\hline $\begin{array}{l}\text { Chakravarthi } \text { et al., 2016a }- \text { TCM199 }(1 \mu \mathrm{g} / \mathrm{ml} \mathrm{T} 4,2.5 \\
\mu \mathrm{g} / \mathrm{ml} \mathrm{FSH}, 10 \mathrm{ng} / \mathrm{ml} \text { IGF-I and } 1 \mathrm{mIU} / \mathrm{ml} \mathrm{GH})\end{array}$ & econdary - Isolated 2D (6 days) & $\begin{array}{l}\text { Quantitative expression of CX32 and CX43 genes in cumulus cells and oocytes at different follicular stages (in vivo vs. } \\
\text { in vitro) - In vitro follicle culture } \downarrow \text { expression of CX32 and CX43. }\end{array}$ \\
\hline $\begin{array}{l}\text { Chakravarthi } \text { et al., 2016b - TCM199 }(1 \mu \mathrm{g} / \mathrm{ml} \mathrm{T} 4,2.5 \\
\mu \mathrm{g} / \mathrm{ml} \text { FSH, } 10 \mathrm{ng} / \mathrm{ml} \mathrm{IGF-I,} 1 \mathrm{mIU} / \mathrm{ml} \mathrm{GH})\end{array}$ & Secondary - Isolated 2D (6 days) & $\begin{array}{l}\text { Quantitative expression of } C C N B 1 \text { and } C C N D 1 \text { genes in cumulus cells and oocytes at different follicular stages (in } \\
\text { vivo vs. in vitro) - In vitro follicle culture unbalanced the expression pattern of CCNB1 and CCND1 }\end{array}$ \\
\hline $\begin{array}{l}\text { Sadeghnia et al., } 2016 \text { - } \alpha \text {-MEM }(10 \mu \mathrm{g} / \mathrm{ml} \text { INS, } 5.5 \mu \mathrm{g} / \mathrm{ml} \\
\text { TRAN, } 5.5 \mathrm{ng} / \mathrm{ml} \mathrm{SEL,} 3 \mathrm{mg} / \mathrm{ml} \mathrm{BSA}, 100 \mathrm{ng} / \mathrm{ml} \mathrm{FSH,} 100 \\
\mathrm{ng} / \mathrm{ml} \text { GDF- } 9,50 \mu \mathrm{g} / \mathrm{ml} \mathrm{AA})\end{array}$ & $\begin{array}{l}\text { Primordial and primary - In } \\
\text { situ }(8 \text { days }) \text { and Isolated 3D }(8 \\
\text { days) }\end{array}$ & $\begin{array}{l}0.5,1 \text { or } 2 \% \text { alginate (ovarian tissue and isolated follicles) - Alginate encapsulation } \downarrow \text { number of secondary follicles. } \\
\text { Encapsulation } \uparrow \text { follicle growth }\end{array}$ \\
\hline $\begin{array}{l}\text { Kamalamma et al., 2016 } \\
\text { supplemented by } 1 \mu \mathrm{g} / \mathrm{ml} \text { TCM199 } 4,2.5 \mu \mathrm{g} / \mathrm{ml} \text { FSH, } 10 \mathrm{ng} / \mathrm{ml} \\
\text { IGF-I, } 1 \mathrm{mIU} / \mathrm{ml} \mathrm{GH} \text { ) }\end{array}$ & econdary - Isolated 2D (6 days) & $\begin{array}{l}\text { 0-1000 } \mathbf{~ g} / \mathbf{m l} \text { leptin and } 10 \mathrm{ng} / \mathrm{ml} \text { human leptin vs. } 10 \mathrm{ng} / \mathrm{ml} \text { ovine leptin }-\uparrow \text { growing follicles, diameter, antrum } \\
\text { formation and oocyte maturationcin TCM199 with supplementation and } 10 \mathrm{ng} / \mathrm{ml} \text { human or ovine leptin. }\end{array}$ \\
\hline $\begin{array}{l}\text { Kona et al., } 2016 \text { - TCM199 }(1 \mu \mathrm{g} / \mathrm{ml} \mathrm{T} 4,2.5 \mu \mathrm{g} / \mathrm{ml} \mathrm{FSH,} \\
10 \mathrm{ng} / \mathrm{ml} \mathrm{IGF}-\mathrm{I}, 1 \mathrm{mIU} / \mathrm{ml} \mathrm{GH})\end{array}$ & Secondary - Isolated 2D (6 days) & $\begin{array}{l}\text { Quantitative expression of GDF9 and BMP15 genes in cumulus cells and oocytes at different follicular stages (in vivo } \\
\text { vs. in vitro) -In vitro follicle culture altered the stage-specific changes in the expression of GDF9 and BMP15 }\end{array}$ \\
\hline $\begin{array}{l}\text { Lins et al., } 2107 \text { - } \alpha \text {-MEM(10 } \mathrm{ng} / \mathrm{ml} \mathrm{INS}, 3 \mathrm{mg} / \mathrm{ml} \mathrm{BSA,} 2 \\
\mathrm{mM} \text { GLUT, } 2 \mathrm{mM} \text { HYPO) }\end{array}$ & $\begin{array}{llll}\begin{array}{l}\text { Secondary } \\
\text { (12days) }\end{array} & - & \text { Isolated } & 2 \mathrm{D} \\
\end{array}$ & 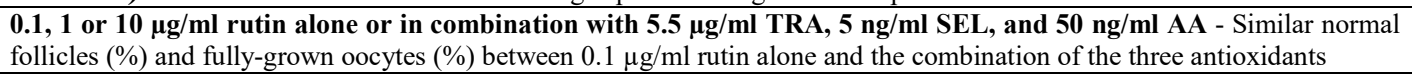 \\
\hline $\begin{array}{l}\text { Nandi } \text { et al., } 2017-\alpha \text {-MEM (1\% ITS, } 3 \mathrm{mg} / \mathrm{ml} \text { BSA, } 2 \mathrm{mM} \\
\text { GLUT, } 2 \mathrm{mM} \text { HYPO, } 7 \mu \mathrm{g} / \mathrm{ml} \text { FSH and } 0.23 \mathrm{mM} \text { PYR })\end{array}$ & $\begin{array}{l}\text { Secondary - Isolated 2D ( } 7 \text { and } \\
14 \text { days })\end{array}$ & BHB - Minimum concentrations to impair follicle function. \\
\hline $\begin{array}{l}\text { Mbemya } \text { et al., 2017 - } \alpha \text {-MEM }(10 \mu \mathrm{g} / \mathrm{ml} \text { INS, } 5.5 \mu \mathrm{g} / \mathrm{ml} \\
\text { TRAN, } 5 \mathrm{ng} / \mathrm{ml} \mathrm{SEL}, 1.25 \mathrm{mg} / \mathrm{ml} \text { BSA, } 2 \mathrm{mM} \text { GLUT, } 2 \\
\mathrm{mM} \text { HYPO) }\end{array}$ & $\begin{array}{l}\text { Primordial and primary - In } \\
\text { situ ( } 7 \text { days) }\end{array}$ & $\begin{array}{l}50 \mathrm{ng} / \mathrm{ml} \mathrm{FSH} \mathrm{vs.} \mathbf{0 . 3}, \mathbf{1 . 2 5} \text {, or } 5 \mathrm{mg} / \mathrm{ml} \text { J.insularis. } 300 \mu \mathrm{g} / \mathrm{ml} \text { Anethole with } 0.3 \mathrm{mg} / \mathrm{ml} \text { J.insularis, or } 50 \mathrm{ng} / \mathrm{ml} \mathrm{FSH} \mathrm{-} \\
0.3 \mathrm{ng} / \mathrm{ml} \text { J.insularis maintained follicle morphology. }\end{array}$ \\
\hline
\end{tabular}


In comparison with the other two species reviewed in the present article, the caprine has been by far the most studied species regarding folliculogenesis in vitro in the last years (Table 3). A few 2- to 16-cell embryos have been produced from secondary follicles cultured in vitro in the isolated form (Saraiva et al., 2010; Silva et al., 2014), and even one has reached the morula stage after IVF (Magalhães et al., 2011). Despite that, oocyte maturation and embryo production rates are still low when compared to oocytes grown in vivo. In this regard, wishing to understand and improve conditions for successful IVFC in goats, different substances and/or culture systems have been tested on different follicular categories.

Isolated secondary follicles $(\sim 200 \mu \mathrm{m})$ improved their growth rates in the presence of increasing concentrations of FSH (100 ng/ml D0-D6; $500 \mathrm{ng} / \mathrm{ml}$ D6-D12; $1000 \mathrm{ng} / \mathrm{ml}$ D12-D18) alone (Saraiva et al., 2011) or in combination with LH and/or EGF (Saraiva et al., 2010; Silva et al., 2013). Moreover, sequential increasing FSH concentrations stimulated oocyte meiotic resumption to the GVBD stage when added alone (Saraiva et al., 2011) or in combination with IGF-I (Magalhães-Padilha et al., 2012 b), while a low number of oocytes were able to reach the MII stage when sequential FSH was combined with: insulin (Chaves et al., 2012), IGF-II (Duarte et al., 2013), EGF (Silva et al., 2013), LH and EGF (Saraiva et al., 2010), GH (Magalhães et al., 2011), and VEGF (Araújo et al., 2011; Silva et al., 2014). Moreover, a few of these MII oocytes were fertilized and continued further development into 8-cell embryo (Silva et al., 2014), 16-cell embryo (Saraiva et al., 2010), and morula (Magalhães et al., 2011), which is the latest embryo developmental stage attained so far. Insulin and FSH are present in almost every culture medium for IVFC, although their concentration when combined is still focus of discussion (DipazBerrocal et al., 2017; Paes et al., 2018). It has been suggested that $10 \mathrm{ng} / \mathrm{ml}$ insulin, a lower concentration than that which comes in the ITS composition, could be more efficient in promoting meiotic resumption in the presence sequential FSH (Chaves et al., 2012). However, some authors described that in the presence of either GH or VEGF, a high insulin concentration (10 $\mu \mathrm{g} / \mathrm{ml}$ ) combined with fixed $100 \mathrm{ng} / \mathrm{ml} \mathrm{FSH}$ instead of sequential FSH can improve oocyte developmental competence (Ferreira et al., 2016; Silva et al., 2017b); and also stimulate antrum formation in the presence of phytohemagglutinin (PHA) (Cunha et al., 2013). Conversely, Ferreira et al. (2018) showed no positive effect of the association of high insulin and fixed FSH. This fact might be due to the source of FSH since Ferreira et al. (2018) used recombinant human FSH while most studies used recombinant bovine FSH. Conversely, fixed $10 \mathrm{mIU} / \mathrm{ml}$ human FSH improved oocyte meiotic resumption when compared to sequential bovine FSH (Rocha et al., 2014). Even the base medium itself can influence the follicular response to FSH and insulin. Hence, Amburana cearensis (Amb) ethanolic extract $(0.2 \mathrm{mg} / \mathrm{ml})$ base medium promoted higher follicle daily growth rate in the presence of sequential FSH and low insulin concentration than $\alpha$-minimum essential medium ( $\alpha$-MEM) (Gouveia et al., 2016). supplement may also depend on follicular category since it has been shown that PFs and early antral follicles (EAFs) behave differently under the same culture conditions (Cadenas et al., 2017). Thus, human FSH increased follicle and oocyte diameters of EAFs $(\sim 350 \mu \mathrm{m})$ but did not affect PFs (Ferreira et al., 2018). Likewise, unlike PFs, EAFs have shown the greatest MII rate described so far from in vitro grown oocytes $(46.2 \%$ calculated out of the total number of cultured follicles) in response to $\mathrm{GH}$ added to a medium with low insulin and no FSH (Cadenas et al., 2018). Also, Cadenas et al. (2018) were able to identify some non-invasive signs for the efficiency of IVFC for EAFs: follicle daily growth $\geq 6.1 \mu \mathrm{m}$, follicle diameter $\geq 600.1 \mu \mathrm{m}$, and oocyte diameter $\geq 120.1 \mu \mathrm{m}$.

Besides culture media composition, many other factors have shown to affect the development of isolated early stage follicles such as the reproductive age of the ovary donor (prepubertal vs. adult), culture period, base media, and culture system (2D vs. 3D). Hence, PFs from prepubertal goats have reached the antral stage, but contrary to PFs from adult goats, were not able to produce MII oocytes after 18 days of IVFC (Amin et al., 2013; Silva et al., 2014) regardless the culture system, i.e., $2 \mathrm{D}$ vs $0.5 \%$ alginate (3D) (Silva et al., 2014). However, Brito et al. (2014) related a positive effect of a lower matrix stiffness $(0.25 \%$ alginate $)$ on follicle growth and oocyte meiotic resumption when compared to $0.5 \%$ alginate and $2 \mathrm{D}$ system. Furthermore, the coculture of $5 \mathrm{PFs}$ per alginate bead stimulated follicle growth, and also a new matrix composed by $12.5 \mathrm{mg} / \mathrm{ml}$ fibrinogen and $0.125 \%$ alginate (fibrinalginate) improved oocyte meiotic resumption when compared to $0.25 \%$ alginate (Brito et al., 2016).

The suitable culture period for IVFC in the isolated form is still a matter of debate. Even though 18 days is the most commonly used for large secondary follicles (Ferreira et al., 2016; Silva et al., 2017b), it seems that this follicular category may benefit from an extended culture period (30 to 36 days) (Pessoa et al., 2014), while 18 days has been described as the most suitable culture period for EAFs (Cadenas et al., 2018).

Primordial and primary follicles have been usually cultured in situ. Within this culture system, several authors have reported the stimulation of follicle activation, and follicle and oocyte growth after a long-term culture (16 days) in the presence of: FSH during the first half (D0D8) followed by either GH (Magalhães-Padilha et al., 2012a) or fibroblast growth factor-10 (FGF-10) during the second half (D8-D16) of the culture period (Almeida et al., 2015); KL during the first half and FSH during the second half of the culture (Lima et al., 2012); and also, FSH and IGF-I throughout the entire culture period, which turned up in increasing the percentage of secondary follicles (28\%) (Magalhaes-Padilha et al., 2012). Other substances added for a shorter culture time (6 to 7 days) have also shown to exert a positive effect on follicle activation, survival and growth such as: the interaction between melatonin and FSH (Rocha et al., 2013); EGF (Lopes et al., 2015); KL (Faustino et al., 2013); and Concavalin A (Con A) (Portela et al., 2014). On the other hand, Keratinocyte growth factor-1 (KGF-1), also known as FGF-7, did not have a positive impact on early folliculogenesis in vitro (Faustino et al., 2013). 
Table 3. Chronological advances in in vitro culture of caprine preantral follicles*

\begin{tabular}{|c|c|c|}
\hline References/Base medium & Follicle Category/Culture system & Tested compounds/Main findings \\
\hline $\begin{array}{l}\text { Saraiva } \text { et al., } 2010-\alpha-M E M(3 \mathrm{mg} / \mathrm{ml} \mathrm{BSA}, 10 \mu \mathrm{g} / \mathrm{ml} \mathrm{INS}, \\
5.5 \mu \mathrm{g} / \mathrm{ml} \text { TRAN, } 5 \mathrm{ng} / \mathrm{ml} \text { SEL, } 2 \mathrm{mM} \text { GLUT, } 2 \mathrm{mM} \text { HYPO, } 50 \\
\mu \mathrm{g} / \mathrm{ml} \text { AA, Sequential FSH) }\end{array}$ & Secondary - Isolated 2D (18 days) & $\begin{array}{l}50 \text { or } 100 \mathbf{~ n g} / \mathbf{m l} \mathbf{~ L H} \text { and 50, or } 100 \mathbf{~ n g} / \mathbf{m l ~ E G F} \text { alone or associated - LH+EGF } \uparrow \text { follicle growth } \\
\text { and oocyte meiotic resumption. } 2 \text { embryos (8- and 16-cell) in } 100 \mathrm{ng} / \mathrm{ml} \text { LH+EGF }\end{array}$ \\
\hline $\begin{array}{l}\text { Saraiva et al., 2011 - } \alpha \text {-MEM }(1.25 \mathrm{mg} / \mathrm{ml} \mathrm{BSA,} 10 \mu \mathrm{g} / \mathrm{ml} \text { INS, } \\
5.5 \mu \mathrm{g} / \mathrm{ml} \text { TRAN, } 5 \mathrm{ng} / \mathrm{ml} \text { SEL, } 2 \mathrm{mM} \text { GLUT, } 2 \mathrm{mM} \text { HYPO, } 50 \\
\mu \mathrm{g} / \mathrm{ml} \text { AA) }\end{array}$ & Secondary - Isolated 2D (18 days) & $\begin{array}{l}\text { Fixed }(100, \text { or } 1000 \mathrm{ng} / \mathrm{ml}) \text {, Sequential FSH - Sequential FSH } \downarrow \text { extrusion, } \uparrow \text { antrum formation and } \\
\text { oocyte meiotic resumption. }\end{array}$ \\
\hline $\begin{array}{l}\text { Magalhães et al., } 2011 \text { - } \alpha \text {-MEM ( } 3 \mathrm{mg} / \mathrm{ml} \text { BSA, } 10 \mu \mathrm{g} / \mathrm{ml} \text { INS, } \\
5.5 \mu \mathrm{g} / \mathrm{ml} \text { TRAN, } 5 \mathrm{ng} / \mathrm{ml} \mathrm{SEL}, 2 \mathrm{mM} \text { GLUT, } 2 \mathrm{mM} \text { HYPO, } 50 \\
\mu \mathrm{g} / \mathrm{ml} \text { AA, Sequential FSH) }\end{array}$ & Secondary - Isolated 2D (18 days) & $\begin{array}{l}10 \text { or } 50 \mathrm{ng} / \mathrm{ml} \mathrm{GH}-50 \mathrm{ng} / \mathrm{ml} \mathrm{GH} \uparrow \text { antrum formation and oocyte meiotic resumption. } 1 \text { morula } \\
\text { from } 50 \mathrm{ng} / \mathrm{ml} \mathrm{GH}\end{array}$ \\
\hline $\begin{array}{l}\text { Araújo et al., } 2011 \text { - } \alpha \text {-MEM ( } 3 \mathrm{mg} / \mathrm{ml} \text { BSA, } 10 \mu \mathrm{g} / \mathrm{ml} \text { INS, } 5.5 \\
\mu \mathrm{g} / \mathrm{ml} \text { TRAN, } 5 \mathrm{ng} / \mathrm{ml} \text { SEL, } 2 \mathrm{mM} \text { GLUT, } 2 \mathrm{mM} \text { HYPO, } 50 \\
\mu \mathrm{g} / \mathrm{ml} \text { AA, Sequential FSH) }\end{array}$ & Secondary - Isolated 2D (18 days) & 10 or $100 \mathrm{ng} / \mathrm{ml}$ VEGF - $100 \mathrm{ng} / \mathrm{ml}$ VEGF $\uparrow$ oocyte meiotic resumption \\
\hline $\begin{array}{l}\text { Chaves } \text { et al., } 2012 \text { - } \alpha \text {-MEM ( } 3 \mathrm{mg} / \mathrm{ml} \text { BSA, } 5.5 \mu \mathrm{g} / \mathrm{ml} \text { TRAN, } \\
5 \mathrm{ng} / \mathrm{ml} \text { SEL, } 2 \mathrm{mM} \text { GLUT, } 2 \mathrm{mM} \text { HYPO, } 50 \mu \mathrm{g} / \mathrm{ml} \mathrm{AA})\end{array}$ & Secondary - Isolated 2D (18 days) & $\begin{array}{l}5 \text { or } 10 \mathrm{ng} / \mathrm{ml} \text {, or } 10 \mu \mathrm{g} / \mathrm{ml} \text { insulin alone or in association to sequential }-10 \mathrm{ng} / \mathrm{ml} \text { insulin with } \\
\text { sequential FSH } \uparrow \text { oocyte meiotic resumption }\end{array}$ \\
\hline $\begin{array}{l}\text { Lima et al., 2012 - } \alpha \text {-MEM }(1.25 \mathrm{mg} / \mathrm{ml} \text { BSA, } 10 \mu \mathrm{g} / \mathrm{ml} \text { INS, } \\
5.5 \mu \mathrm{g} / \mathrm{ml} \text { TRAN, } 5 \mathrm{ng} / \mathrm{ml} \mathrm{SEL}, 2 \mathrm{mM} \text { GLUT, } 2 \mathrm{mM} \text { HYPO, } 50 \\
\mu \mathrm{g} / \mathrm{ml} \mathrm{AA})\end{array}$ & Primordial and primary - In situ (16 days) & $\begin{array}{l}\mathbf{5 0} \mathbf{~ n g} / \mathbf{m l ~ K L} \text { and } \mathbf{5 0} \mathbf{~ n g} / \mathbf{m l ~ F S H} \text { alone or in different combinations - KL (D0-D8)/FSH(D8-D16) } \\
\text { 个activation and growth }\end{array}$ \\
\hline $\begin{array}{l}\text { Magalhães-Padilha } \text { et al., } 2012 \text { - } \alpha \text {-MEM }(1.25 \mathrm{mg} / \mathrm{ml} \text { BSA, } \\
10 \mu \mathrm{g} / \mathrm{ml} \text { INS, } 5.5 \mu \mathrm{g} / \mathrm{ml} \text { TRAN, } 5 \mathrm{ng} / \mathrm{ml} \mathrm{SEL}, 2 \mathrm{mM} \text { GLUT, } 2 \\
\mathrm{mM} \text { HYPO, } 50 \mu \mathrm{g} / \mathrm{ml} \text { AA) }\end{array}$ & Primordial and primary - In situ (16 days) & $\begin{array}{l}50 \mathbf{~ n g} / \mathbf{m l ~ I G F - I ~ a n d ~} \mathbf{5 0} \mathbf{~ n g} / \mathbf{m l} \text { FSH alone, or in different combinations - IGF-I+FSH 个activation } \\
\text { and growth, number of secondary follicles }\end{array}$ \\
\hline $\begin{array}{l}\text { Magalhães-Padilha et al., 2012a - } \alpha \text {-MEM }(1.25 \mathrm{mg} / \mathrm{ml} \text { BSA, } \\
10 \mu \mathrm{g} / \mathrm{ml} \text { INS, } 5.5 \mu \mathrm{g} / \mathrm{ml} \text { TRAN, } 5 \mathrm{ng} / \mathrm{ml} \mathrm{SEL}, 2 \mathrm{mM} \text { GLUT, } 2 \\
\mathrm{mM} \text { HYPO, } 50 \mu \mathrm{g} / \mathrm{ml} \mathrm{AA})\end{array}$ & Primordial and primary - In situ (16 days) & $\begin{array}{l}10 \mathrm{ng} / \mathrm{ml} \mathrm{GH} \text { and } 50 \mathrm{ng} / \mathrm{ml} \mathrm{FSH} \mathrm{alone,} \mathrm{or} \mathrm{in} \mathrm{different} \mathrm{combinations} \mathrm{-} \mathrm{FSH} \mathrm{(D0-D8)/GH} \mathrm{(D8-D16)} \\
\uparrow \text { follicle morphology, viability, activation, growth and secondary follicles }\end{array}$ \\
\hline $\begin{array}{l}\text { Magalhães-Padilha } \text { et al., 2012b - } \alpha \text {-MEM ( } 3 \mathrm{mg} / \mathrm{ml} \mathrm{BSA,} 10 \\
\mu \mathrm{g} / \mathrm{ml} \mathrm{INS}, 5.5 \mu \mathrm{g} / \mathrm{ml} \text { TRAN, } 5 \mathrm{ng} / \mathrm{ml} \mathrm{SEL}, 2 \mathrm{mM} \text { GLUT, } 2 \\
\mathrm{mM} \text { HYPO, } 50 \mu \mathrm{g} / \mathrm{ml} \text { AA, Sequential FSH) }\end{array}$ & Secondary - Isolated 2D (18 days) & 50 or $100 \mathrm{ng} / \mathrm{ml}$ IGF-I - IGF-I $\uparrow$ oocyte meiotic resumption. $50 \mathrm{ng} / \mathrm{ml}$ IGF-I $\uparrow$ antrum formation \\
\hline $\begin{array}{l}\text { Cunha } \text { et al., } 2013 \text { - } \alpha \text {-MEM ( } 3 \mathrm{mg} / \mathrm{ml} \text { BSA, } 10 \mu \mathrm{g} / \mathrm{ml} \text { INS, } 5.5 \\
\mu \mathrm{g} / \mathrm{ml} \text { TRAN, } 5 \mathrm{ng} / \mathrm{ml} \mathrm{SEL}, 2 \mathrm{mM} \text { GLUT, } 2 \mathrm{mM} \text { HYPO, } 50 \\
\mu \mathrm{g} / \mathrm{ml} \mathrm{AA}, 100 \mathrm{ng} / \mathrm{ml} \mathrm{FSH})\end{array}$ & Secondary - Isolated 2D (18 days) & $\begin{array}{l}\text { 1, 10, 50, } 100 \text { or } 200 \mu \mathrm{g} / \mathrm{ml} \text { PHA-10 } \mu \mathrm{g} / \mathrm{ml} \text { PHA maintained follicle ultrastructure and } \uparrow \text { antrum } \\
\text { formation }\end{array}$ \\
\hline $\begin{array}{l}\text { Duarte et al., } 2013 \text { - } \alpha \text {-MEM }(3 \mathrm{mg} / \mathrm{ml} \mathrm{BSA,} 10 \mathrm{ng} / \mathrm{ml} \text { INS, } 5.5 \\
\mu \mathrm{g} / \mathrm{ml} \text { TRAN, } 5 \mathrm{ng} / \mathrm{ml} \mathrm{SEL}, 2 \mathrm{mM} \text { GLUT, } 2 \mathrm{mM} \text { HYPO, } 50 \\
\mu \mathrm{g} / \mathrm{ml} \text { AA) }\end{array}$ & Secondary - Isolated 2D (18 days) & $\begin{array}{l}20 \text { or } 50 \mathrm{ng} / \mathrm{ml} \text { IGF-II, or sequential FSH - } 20 \mathrm{ng} / \mathrm{ml} \text { IGF-II alone or in associated to sequential FSH } \\
\text { 个 oocyte meiotic resumption }\end{array}$ \\
\hline $\begin{array}{l}\text { Faustino } \text { et al., } 2013 \text { - } \alpha \text {-MEM }(1.25 \mathrm{mg} / \mathrm{ml} \text { BSA, } 10 \mu \mathrm{g} / \mathrm{ml} \\
\text { INS, } 5.5 \mu \mathrm{g} / \mathrm{ml} \text { TRAN, } 5 \mathrm{ng} / \mathrm{ml} \text { SEL, } 2 \mathrm{mM} \text { GLUT, } 2 \mathrm{mM} \\
\mathrm{HYPO}, 50 \mu \mathrm{g} / \mathrm{ml} \text { AA })\end{array}$ & Primordial and primary - In situ (7 days) & $\begin{array}{l}1 \mathrm{ng} / \mathrm{ml} \text { KGF-1 and } 50 \mathrm{ng} / \mathrm{ml} \text { KL alone or in combination - } 50 \mathrm{ng} / \mathrm{ml} \mathrm{KL} \text { alone } \uparrow \text { activation and } \\
\text { growth }\end{array}$ \\
\hline $\begin{array}{l}\text { Rocha et al., 2013 - } \alpha \text {-MEM }(1.25 \mathrm{mg} / \mathrm{ml} \text { BSA, } 10 \mu \mathrm{g} / \mathrm{ml} \text { INS, } \\
5.5 \mu \mathrm{g} / \mathrm{ml} \text { TRAN, } 5 \mathrm{ng} / \mathrm{ml} \text { SEL, } 2 \mathrm{mM} \text { GLUT, } 2 \mathrm{mM} \text { HYPO, } \\
0.23 \mathrm{mM} \text { PYR })\end{array}$ & Primordial and primary - In situ (7 days) & $\begin{array}{l}\mathbf{1 0 0}, \mathbf{2 5 0}, \mathbf{5 0 0}, \text { or } \mathbf{1 0 0 0} \mathbf{p M} \text { melatonin, and } \mathbf{5 0} \mathrm{ng} / \mathbf{m l} \mathbf{F S H} \text {, alone or in combination - } 1000 \mathrm{pM} \\
\text { melatonin }+50 \mathrm{ng} / \mathrm{ml} \mathrm{FSH} \uparrow \text { follicular and oocyte diameters }\end{array}$ \\
\hline $\begin{array}{l}\text { Silva et al., } 2013 \text { - } \alpha \text {-MEM ( } 3 \mathrm{mg} / \mathrm{ml} \text { BSA, } 10 \mu \mathrm{g} / \mathrm{ml} \text { INS, } 5.5 \\
\mu \mathrm{g} / \mathrm{ml} \text { TRAN, } 5 \mathrm{ng} / \mathrm{ml} \text { SEL, } 2 \mathrm{mM} \text { GLUT, } 2 \mathrm{mM} \text { HYPO, } 50 \\
\mu \mathrm{g} / \mathrm{ml} \text { AA, Sequential FSH) }\end{array}$ & Secondary - Isolated 2D (18 days) & $\begin{array}{l}\text { 50 or } 100 \mathrm{ng} / \mathrm{ml} \text { EGF - EGF } \uparrow \text { follicle daily growth rate. } 50 \mathrm{ng} / \mathrm{ml} \text { EGF } \boldsymbol{\uparrow} \text { oocyte meiotic } \\
\text { resumption }\end{array}$ \\
\hline Amin et al., 2013 - Bicarbonate-buffered TCM199 & Secondary - Isolated 2D (6 days) & $\begin{array}{l}\text { Different concentrations and association of T4, FSH, GH, EGF, and IGF-I - All substances alone } \\
\text { or in combination } \uparrow \text { follicle growth and antrum formation. The association } \mathrm{T} 4+\mathrm{FSH}+\mathrm{GH}+\mathrm{EGF} \boldsymbol{\uparrow} \\
\text { extrusion. }\end{array}$ \\
\hline
\end{tabular}




\section{References/Base medium}

Pessoa et al., 2014 - $\alpha$-MEM ( $3 \mathrm{mg} / \mathrm{ml}$ BSA, $10 \mathrm{ng} / \mathrm{ml}$ INS, 5.5 $\mu \mathrm{g} / \mathrm{ml}$ TRAN, $5 \mathrm{ng} / \mathrm{ml}$ SEL, $2 \mathrm{mM}$ GLUT, $2 \mathrm{mM}$ HYPO, 50 $\mu \mathrm{g} / \mathrm{ml} \mathrm{AA}$, Sequential FSH and $50 \mathrm{ng} / \mathrm{ml} \mathrm{GH})$

Portela et al., 2014 - $\alpha$-MEM $(1.25 \mathrm{mg} / \mathrm{ml}$ BSA, $10 \mu \mathrm{g} / \mathrm{ml}$ INS,

$5.5 \mu \mathrm{g} / \mathrm{ml}$ TRAN, $5 \mathrm{ng} / \mathrm{ml}$ SEL, $2 \mathrm{mM}$ GLUT, $2 \mathrm{mM}$ HYPO, $0.23 \mathrm{mM}$ PYR)

Silva et al 2014 - $\alpha$-MEM $(3 \mathrm{mg} / \mathrm{ml}$ BSA, $10 \mu \mathrm{g} / \mathrm{ml}$ INS, 5.5 $\mu \mathrm{g} / \mathrm{ml}$ TRAN, $5 \mathrm{ng} / \mathrm{ml}$ SEL, $2 \mathrm{mM}$ GLUT, $2 \mathrm{mM}$ HYPO, 50 $\mu \mathrm{g} / \mathrm{ml} \mathrm{AA}$, Sequential FSH, $100 \mathrm{ng} / \mathrm{ml}$ VEGF, $1 \mathrm{mg} / \mathrm{ml}$ FET

Rocha et al., 2014 - $\alpha$-MEM $(3 \mathrm{mg} / \mathrm{ml}$ BSA, $10 \mu \mathrm{g} / \mathrm{ml}$ INS, 5.5 $\mu \mathrm{g} / \mathrm{ml}$ TRAN, $5 \mathrm{ng} / \mathrm{ml} \mathrm{SEL}, 2 \mathrm{mM}$ GLUT, $2 \mathrm{mM}$ HYPO, 50 $\mu \mathrm{g} / \mathrm{ml} \mathrm{AA}, 100 \mathrm{ng} / \mathrm{ml}$ VEGF, $1 \mathrm{mg} / \mathrm{ml}$ FET)

Brito et al., 2014- $\alpha$-MEM (3 mg/ml BSA, $10 \mu \mathrm{g} / \mathrm{ml}$ INS, 5.5 $\mu \mathrm{g} / \mathrm{ml}$ TRAN, $5 \mathrm{ng} / \mathrm{ml}$ SEL, $2 \mathrm{mM}$ GLUT, $2 \mathrm{mM}$ HYPO, 50 $\mu \mathrm{g} / \mathrm{ml}$ AA, Sequential FSH

Almeida et al., 2015 - $\alpha$-MEM $(1.25 \mathrm{mg} / \mathrm{ml} \mathrm{BSA}, 10 \mu \mathrm{g} / \mathrm{m}$

INS, $5.5 \mu \mathrm{g} / \mathrm{ml}$ TRAN, $5 \mathrm{ng} / \mathrm{ml}$ SEL, 2 mM GLUT, 2 m HYPO, 50 AA)

Lopes et al., 2015 - $\alpha$-MEM (3 mg/ml BSA, $10 \mu \mathrm{g} / \mathrm{ml}$ INS, 5.5

$\mu \mathrm{g} / \mathrm{ml}$ TRAN, $5 \mathrm{ng} / \mathrm{ml}$ SEL, $2 \mathrm{mM}$ GLUT, 2 mM HYPO, 50 Primos $\mu \mathrm{g} / \mathrm{ml} \mathrm{AA})$

Gouveia et al., 2016 - $\alpha$-MEM or different concentrations of Amb extracts

Ferreira et al., 2016 - $\alpha$-MEM $(3 \mathrm{mg} / \mathrm{ml} \mathrm{BSA}, 5.5 \mu \mathrm{g} / \mathrm{ml}$ TRAN, $5 \mathrm{ng} / \mathrm{ml} \mathrm{SEL}, 2 \mathrm{mM}$ GLUT, $2 \mathrm{mM}$ HYPO, $50 \mu \mathrm{g} / \mathrm{m}$ $\mathrm{AA}$ and $50 \mathrm{ng} / \mathrm{ml} \mathrm{GH}$ )

Brito et 2016 - $\alpha$-MEM $(3 \mathrm{mg} / \mathrm{ml}$ BSA, $10 \mu \mathrm{g} / \mathrm{ml}$ INS, 5.5 Brito et al., 2016 - $a$-MEM ( 3 mg/ml BSA, $10 \mu g / n I N S, 5.5$ $\mu \mathrm{g} / \mathrm{ml}$ TRAN, $5 \mathrm{ng} / \mathrm{ml}$ SEL, 2 mM GLUT, 2 mM HYPO, 50 $\mu \mathrm{g} / \mathrm{ml} \mathrm{AA,} \mathrm{Sequential} \mathrm{FS}$

Silva et al., 2017b - $\alpha$-MEM (3 mg/ml BSA, $5.5 \mu \mathrm{g} / \mathrm{ml}$ TRAN $5 \mathrm{ng} / \mathrm{ml} \mathrm{SEL}, 2 \mathrm{mM}$ GLUT, $2 \mathrm{mM}$ HYPO, $50 \mu \mathrm{g} / \mathrm{ml} \mathrm{AA}$ and $100 \mathrm{ng} / \mathrm{ml}$ VEGF

Cadenas et al., 2017 - $\alpha$-MEM $(3 \mathrm{mg} / \mathrm{ml} \mathrm{BSA,} 10 \mathrm{ng} / \mathrm{ml}$ INS, $5.5 \mu \mathrm{g} / \mathrm{ml}$ TRAN, $5 \mathrm{ng} / \mathrm{ml}$ SEL, $2 \mathrm{mM}$ GLUT, $2 \mathrm{mM}$ HYPO and $50 \mu \mathrm{g} / \mathrm{ml} \mathrm{AA}$ )

Ferreira et al., $2018-\alpha$-MEM $(3 \mathrm{mg} / \mathrm{ml}$ BSA, $10 \mu \mathrm{g} / \mathrm{ml}$ INS

$5.5 \mu \mathrm{g} / \mathrm{ml}$ TRAN, $5 \mathrm{ng} / \mathrm{ml} \mathrm{SEL}, 2 \mathrm{mM}$ GLUT, $2 \mathrm{mM}$ HYPO and $50 \mu \mathrm{g} / \mathrm{ml} \mathrm{AA}$

and $50 \mu \mathrm{g} / \mathrm{ml} \mathrm{AA}$ )

Tested compounds/Main findings

Secondary - Isolated 2D (Different days) and 3D (alginate -36 days)

Primordial and primary - In situ (6 days)

Secondary - Isolated 2D and Isolated 3D $(0.5 \%$ lginate -18 days)

Secondary - Isolated $3 \mathrm{D}(0.5 \%$ alginate -18 days $)$

Secondary - Isolated 3D (alginate - 18 days)

Primordial and primary - In situ (16 days)

Primordial and primary - In situ (6 days)

Secondary - Isolated 2D (12 days)

Secondary - Isolated 2D (18 days)

Secondary - Isolated 3D (alginate -18 days)

Secondary - Isolated 2D (18 days)

Secondary and tertiary - Isolated 2D (24 days PFs and 18 days EAFs) 2-cell embryos in 2D and one 8 cell-embryo in 3D.
Different culture periods: 18, 24, 30, 36, or 42 days (2D vs. 3D) - Follicle diameter and oocyte meiotic resumption $\uparrow$ until day 36 . 3D system did not affect oocyte meiotic resumption.

5, 10, 20 or $10 \mu \mathrm{g} / \mathrm{ml}$ Con A. $10 \mu \mathrm{g} / \mathrm{ml}$ Con A and $50 \mathrm{ng} / \mathrm{ml} \mathrm{FSH,} \mathrm{alone} \mathrm{or} \mathrm{in} \mathrm{combination} \mathrm{-} 10$ $\mu \mathrm{g} / \mathrm{ml}$ Con $\mathrm{A}$ and $50 \mathrm{ng} / \mathrm{ml} \mathrm{FSH}$ alone $\uparrow$ activation.

Follicles from Adult Vs. Prepubertal ovaries (2D vs. 3D) - 3D system $\uparrow$ survival and $\downarrow$ oocyte extrusion. Greater follicle and oocyte and meiotic resumption diameter in 2D from adult ovaries. Four

$10 \mathrm{mIU} / \mathrm{mlvhuman}$ FSH Vs. Sequential FSH - $10 \mathrm{mIU} / \mathrm{ml}$ human FSH $\uparrow$ oocyte meiotic resumption.

$\mathbf{0 . 2 5} \%, \mathbf{0 . 5} \%$, or $\mathbf{1} \%$ alginate $-0.5 \%$ alginate maintained better follicle integrity. $0.25 \%$ alginate follicle diameter, daily growth and oocyte meiotic resumption.

$50 \mathrm{ng} / \mathrm{ml} \mathrm{FGF-10}$ and $50 \mathrm{ng} / \mathrm{ml}$ FSH alone, in combination or sequentially - FSH(D0-D8)/FGF-10 (D8-D16) showed $\boldsymbol{\uparrow}$ percentages of normal and growing follicles

$10 \mu \mathrm{g} / \mathrm{ml}$ PHA and $100 \mu \mathrm{g} / \mathrm{ml}$ EGF alone or in combination (Healthy goats Vs. CAEV) - EGF alone $\uparrow$ growth in both healthy and CAEV infected goats

$\alpha$-MEM Vs. Amb extracts (0.1, 0.2 or $0.4 \mathrm{mg} / \mathrm{ml})$. $\alpha$-MEM, $0.2 \mathrm{mg} / \mathrm{ml} \mathrm{Amb} \mathrm{(3} \mathrm{mg/ml} \mathrm{BSA,} 10$ $\mu \mathrm{g} / \mathrm{ml}$ INS, $5.5 \mu \mathrm{g} / \mathrm{ml}$ TRAN, $5 \mathrm{ng} / \mathrm{ml}$ SEL, $2 \mathrm{mM}$ GLUT, $2 \mathrm{mM}$ HYPO, $50 \mu \mathrm{g} / \mathrm{ml} \mathrm{AA)} \mathrm{or}$ sequential FSH - 100ng/ml $\mathbf{m b - D 6}, \mathbf{5 0 0} \mathbf{~ n g} / \mathbf{m l}_{\text {D6-D12 }}$ ) - $\alpha$-MEM and $0.2 \mathrm{mg} / \mathrm{ml}$ Amb similar morphology, antrum formation and follicle diameter

$10 \mathrm{ng} / \mathrm{ml}$ or $10 \mu \mathrm{g} / \mathrm{ml}$ insulin alone or associated to either fixed $100 \mathrm{ng} / \mathrm{ml} \mathrm{FSH}$ or sequential FSH $-10 \mathrm{ng} / \mathrm{ml}$ insulin $\Downarrow$ extrusion. $10 \mu \mathrm{g} / \mathrm{ml}$ insulin $\uparrow$ follicle growth. $100 \mathrm{ng} / \mathrm{ml} \mathrm{FSH} \uparrow$ oocyte meiotic resumption. $10 \mu \mathrm{g} / \mathrm{ml}$ insulin $+100 \mathrm{ng} / \mathrm{ml} \mathrm{FSH} \uparrow$ mean oocyte diameter

Number of follicles per $0.25 \%$ alginate bead and beads per well. 5 follicles/bead in alginate, fibrin-alginate, or hyaluronate) -5 follicles/bead $\uparrow$ diameter. Alginate $\uparrow$ follicle daily growth. Fibrin-alginate $\uparrow$ oocyte meiotic resumption.

$10 \mathrm{ng} / \mathrm{ml}$ or $10 \mu \mathrm{g} / \mathrm{ml}$ insulin alone or associated to either fixed $100 \mathrm{ng} / \mathrm{ml} \mathrm{FSH} \mathrm{or} \mathrm{sequential} \mathrm{-} 10$ $\mu \mathrm{g} / \mathrm{ml}$ insulin $\uparrow$ follicle growth. $10 \mu \mathrm{g} / \mathrm{ml}$ insulin + fixed FSH $\uparrow$ oocyte meiotic resumption.

$50 \mathrm{ng} / \mathrm{ml}$ GH or $100 \mathrm{ng} / \mathrm{ml}$ VEGF alone, in combination or sequentially (Secondary PF vs. Tertiary EAF) $-50 \mathrm{ng} / \mathrm{ml} \mathrm{GH} \uparrow$ oocyte growth and meiotic resumption only in EAFs.

10, 50 or $100 \mathrm{mIU} / \mathrm{ml}$ human FSH (PFs vs. EAFS) - No positive effect of human FSH on PFs. On EAFs:50 mIU/ml human FSH $\uparrow$ follicle and oocyte growth

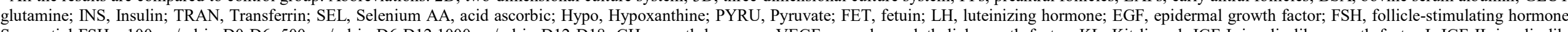

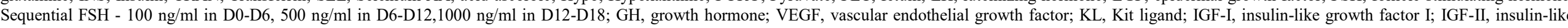

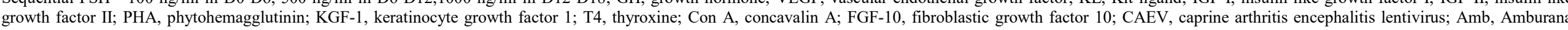
cearensis extract. 
Despite the undeniable progress obtained in goats, oocyte maturation and embryo production rates are still very low compared to oocytes originating from follicles grown in vivo, which must serve for encouraging further research on this topic. Overall, the data generated seem to point out the need to develop future dynamic and customized culture media for IVFC as the differences among follicles regarding their growth rates, and between follicular categories have shown to affect oocyte maturation in vitro.

\section{Future of IVFC: possible new strategies to overcome the current limitations}

To further advance in efficient IVFC methods, some limitations and lack of information need to be overcome. For instance, the in vitro production of a fertilizable oocyte from a primordial follicle requires a long-term culture, which may affect oocyte quality and, consequently embryo prodution. Few studies are reported in animal species other than ruminants, and should be consider when improving culture techniques. It was indicated before that IVC of ovine preantral follicles yield oocytes with normal nuclear-epigenetic maturation (Barboni et al., 2011). However, this study was performed with secondary follicles and not starting from primordial ones, where it was shown in mice that deficiencies at transcriptional and epigenetic levels can occur (Wang et al., 2017). Importantly, IVFC of murine follicles is much shorter than that for large mammals, which can increase the urgence of studies at methylation level. It was demonstrated that apoptosis in murine primary oocytes is mediated by retrotransposon activity (Malki et al., 2014), and the suppresion of this activity is determined by DNA epigenetic modification (Findlay et al., 2015). In other words, methods to measure epigenetic risks, as well as to avoid them during IVFC are still needed. Besides this, preantral follicles are commonly cryopreserved for further in vitro culture. It is well known that the stress caused during exposure to cryoprotectants and the cooling process itself affect important organelles like the endoplasmic reticulum (ER) requiring the possible culture medium enrichment with antioxidants (Brito et al., 2013). Studying the ultrastructure of caprine preantral follicles, it was observed that atretic preantral follicles usually presented damaged ER (Silva et al., 2000).

\section{Final consideration}

The control of the survival, activation and development of ruminant follicles in vitro is hugely complex and involves multiple interactions among extra and intraovarian factors and can be influenced by the type of base culture media, medium replacement regime, type of culture system (2D vs 3D), culture duration, ovarian source (pre-pubertal vs adult), extracellular matrix components, follicular categories (preantral vs early follicles). Unfortunately, these factors do not act on an isolated form, but interact with each other, making the development of IVFC protocols a challenge. Encouraging results have been reported including satisfactory rates of follicle survival, activation, antral formation and the production of fully grown meiotically competent oocytes especially in caprine and ovine species. However, the in vitro embryo production from in vitro grown oocytes is still low. Therefore, improvements in in vitro follicle culture system should be done to improve oocyte quality (oocyte developmental competence) for further production of viable offspring. This fact will allow the future use of a large number of immature oocytes enclosed in PFs in assisted reproductive technologies in humans as well as in others mammalian species.

\section{References}

Almeida AP, Magalhães-Padilha DM, Araújo VR, Costa SL, Chaves RN, Lopes CAP, Donato MAM, Peixoto CA, Campello CC, Junior JB, Figueiredo JR. 2015. Effect of sequential medium with fibroblast growth factor-10 and follicle stimulating hormone on in vitro development of goat preantral follicles. Anim Reprod Sci, 152:32-38.

Amin RU, Chandrashekar Reddy K, Sadasiva Rao K, Raghavender KBP, Teja A, Ramesh T, Arunakumari G. 2013. In vitro culture of goat preantral follicles from fetal ovaries. Small Rumin Res, 115:71-76.

Araújo VR, Silva GM, Duarte ABG, Magalhães DM, Almeida AP, Gonçalves RFB, Bruno JB, Silva TFP, Campello CC, Rodrigues APR, Figueiredo JR. 2011. Vascular endothelial growth factor-A (165) (VEGF-A (165) stimulates the in vitro development and oocyte competence of goat preantral follicles. Cell Tissue Res, 346:273-81.

Araújo V, Gastal M, Wischral A, Figueiredo J, Gastal E. 2014a. In vitro development of bovine secondary follicles in two- and three-dimensional culture systems using vascular endothelial growth factor, insulin-like growth factor-1, and growth hormone. Theriogenology, 82:1246-53.

Araújo VR, Gastal MO, Figueiredo JR, Gastal EL. 2014b. In vitro culture of bovine preantral follicles: a review. Reprod Biol Endocrinol, 12:1-14.

Araújo VR, Gastal MO, Wischral A, Figueiredo JR, Gastal EL. 2015. Long-term in vitro culture of bovine preantral follicles: Effect of base medium and medium replacement methods. Anim Reprod Sci, 161:23-31.

Arunakumari G, Shanmugasundaram N, Rao VH. 2010. Development of morulae from the oocytes of cultured sheep preantral follicles. Theriogenology, 74:884-94.

Barboni B, Russo V, Cecconi S, Curini V, Colosimo A, Garofalo MLA, Capacchietti G, Di Giacinto O, Mattioli M. 2011. In vitro grown sheep preantral follicles yield oocytes with normal nuclear-epigenetic maturation. PLoS One, 6:e27550.

Bertoldo MJ, Duffard N, Bernard J, Frapsauce C, Calais L, Rico C, Mermillod P, Locatelli Y. 2014. Effects of bone morphogenetic protein 4 (BMP4) supplementation during culture of the sheep ovarian 
cortex. Anim Reprod Sci, 149:124-34.

Brito DC, Brito AB, Scalercio SR, Percario S, Miranda MS, Rocha RM, Dizniz JA, Oskam IC, van den Hurk R, Paris MC, Domingues SFS, Santos RR. 2013. Vitamin E-analog trolox prevents endoplasmic reticulum stress in frozen-thawed ovarian tissue of capuchin monkey (Sapajus apella). Cell Tissue Res, 355:471-480.

Brito IR, Silva CMG, Duarte ABG, Lima IMT, Rodrigues GQ, Rossetto R, Sales AD, Lobo CH, Bernuci MP, Rosa-e-Silva ACJS, Campello CC, Xu M, Figueiredo JR. 2014. Alginate hydrogel matrix stiffness influences the in vitro development of caprine preantral follicles. Mol Reprod Dev, 81:636-645.

Brito IR, Silva GM, Sales AD, Lobo CH, Rodrigues GQ, Sousa RF, Moura AAA, Calderón CEM, Bertolini M, Campello CC, Smitz J, Figueiredo JR. 2016. Fibrin-alginate hydrogel supports steroidogenesis, in vitro maturation of oocytes and parthenotes production from caprine preantral follicles cultured in group. Reprod Domest Anim, 51:997-1009.

Cadenas J, Leiva-Revilla J, Vieira LA, Apolloni LB, Aguiar FLN, Alves BG, Lobo CH, Rodrigues APR, Apgar GA, Smitz J, Figueiredo JR, Maside C. 2017. Caprine ovarian follicle requirements differ between preantral and early antral stages after IVC in medium supplemented with GH and VEGF alone or in combination. Theriogenology, 87:323-332.

Cadenas J, Maside C, Ferreira ACA, Vieira LA, Leiva-Revilla J, Paes VM, Alves BG, Brandão FZ, Rodrigues APR, Wheeler MB, Figueiredo JR. 2018. Relationship between follicular dynamics and oocyte maturation during in vitro culture as a non-invasive sign of caprine oocyte meiotic competence. Theriogenology, 107:95-103.

Castro S, Carvalho A, Silva C, Santos F, Campello CC, Figueiredo JR, Rodrigues APR. 2014. Fresh and vitrified bovine preantral follicles have different nutritional requirements during in vitro culture. Cell Tissue Bank, 15:591-601.

Chakravarthi VP, Kona SSR, Kumar AVNS, Bhaskar M, Rao VH. 2016a. Quantitative patterns of expression of gap junction genes during in vivo or in vitro development of ovarian follicles in sheep. Small Rumin Res, 143:35-42.

Chakravarthi VP, Kona SSR, Kumar AVNS, Bhaskara M, Rao VH. 2016b. Stage specific expression of cell cycle genes during in vivo or in vitro development of ovarian follicles in sheep. Small Rumin Res. 143:1-7.

Chaves RN, Duarte ABG, Rodrigues GQ, Celestino JJH, Silva GM, Lopes CAP, Almeida AP, Donato MAM, Peixoto CA, Moura AAA, Lobo CH, Locatelli Y, Mermillod P, Campello CC, Figueiredo JR. 2012. The effects of insulin and follicle-stimulating hormone (FSH) during in vitro development of ovarian goat preantral follicles and the relative mRNA expression for insulin and FSH receptors and cytochrome P450 aromatase in cultured follicles. Biol Reprod, 87:69.

Cunha EV, Costa JJN, Rossi RODS, Silva AWB, Passos JRS, Portela AMLR, Pereira DCST, Donato MAM, Campello CC, Saraiva MVA, Peixoto CA,
Silva JRV, Santos RP. 2013. Phytohemagglutinin improves the development and ultrastructure of in vitrocultured goat (Capra hircus) preantral follicles. Brazilian J Med Biol Res, 46:245-252.

Dipaz-Berrocal DJ, Sa N, Guerreiro D, Celestino JJH, Leiva-Revilla J, Alves KA, Santos RR, Cibin FWS, Rodrigues APR, Figueiredo JR. 2017. Refining insulin concentrations in culture medium containing growth factors BMP15 and GDF9: An in vitro study of the effects on follicle development of goats. Anim Reprod Sci, 185:118-127.

Duarte ABG, Araújo VR, Chaves RN, Silva GM, Luz VB, Haag KT, Magalhães-Padilha DM, Almeida AP, Lobo CH, Campello CC, Figueiredo JR. 2013. Insulinlike growth factor II (IGF-II) and follicle stimulating hormone $(\mathrm{FSH})$ combinations can improve the in vitro development of grown oocytes enclosed in caprine preantral follicles. Growth Horm IGF Res, 23:37-44.

Eppig JJ, Schroeder AC. 1989. Capacity of mouse oocytes from preantral follicles to undergo embryogenesis and development to live young after growth, maturation, and fertilization in vitro. Biol Reprod, 41:268-276.

FAO. [homepage on the internet]. 2010. Food and Agriculture Organization of the United Nations. Available: http://www.faostat.fao.org. Accessed in September 29th. 2018.

Faustino LR, Lima IMT, Carvalho AA, Silva CMG, Castro SV, Lobo CH, Lucci CM, Campello CC, Figueiredo JR, Rodrigues APR. 2013. Interaction between keratinocyte growth factor-1 and kit ligand on the goat preantral follicles cultured in vitro. Small Rumin Res, 114:112-119.

Ferreira ACA, Sá NAR, Guerreiro DD, Vieira HHC, Leiva-Revilla J, Lobo CH, Maside C, Araújo VR, Apgar GA, Brandão FZ, Figueiredo JR, Campello CC. 2016. Balance of insulin and FSH concentrations improves the in vitro development of isolated goat preantral follicles in medium containing GH. Anim Reprod Sci, 165:1-10.

Ferreira ACC, Cadenas J, Sá NAR, Correia HHV, Guerreiro DD, Lobo CH, Alves BG, Maside C, Gastal EL, Rodrigues APR, Figueiredo JR. 2018. In vitro culture of isolated preantral and antral follicles of goats using human recombinant FSH: Concentrationdependent and stage-specific effect. Anim Reprod Sci, 196:120-129. doi: 10.1016/j.anireprosci.2018.07.004.

Figueiredo JR, Rodrigues AP, Silva JR, Santos RR. 2011. Cryopreservation and in vitro culture of caprine preantral follicles. Reprod Fertil Dev, 23:40-47.

Figueiredo JR, Lima LF, Silva JR, Santos RR. 2018. Control of growth and development of preantral follicle: insights from in vitro culture. Anim Reprod, 15:648-659. Findlay JK, Hutt KJ, Hickey M, Anderson RA. 2015. How is the number of primordial follicles in the ovarian reserve established? Biol Reprod, 93:1-7.

Gouveia BB, Macedo TJS, Santos JMS, Barberino RS, Menezes VG, Müller MC, Almeida JRGS, Figueiredo JR, Matos MHT. 2016. Supplemented base medium containing Amburana cearensis associated with FSH improves in vitro development of isolated goat preantral follicles. Theriogenology, 86:1275-1284. 
Green LJ, Shikanov A. 2016. In vitro culture methods of preantral follicles. Theriogenology, 86:229-238.

Hirao Y, Itoh T, Shimizu M, Iga K, Aoyagi K, Kobayashi M, Kacchi M, Hoshi H, Takenouchi N. 2004. In vitro growth and development of bovine oocyte-granulosa cell complexes on the flat substratum: effects of high polyvinylpyrrolidone concentration in culture medium. Biol Reprod, 70, 83-91.

Jimenez CR, Araújo VR, Penintente-Filho JM, Azevedo JL, Silveira RG, Torres CAA. 2016. The base medium affects ultrastructure and survival of bovine preantral follicles cultured in vitro. Theriogenology, 85:1019-1029.

Jorssen EPA, Langbeen A, Fransen E, Martinez EL, Leroy JLMR, Bols PEJ. 2014. Monitoring preantral follicle survival and growth in bovine ovarian biopsies by repeated use of neutral red and cultured in vitro under low and high oxygen tension. Theriogenology, 82:387-395.

Kamalamma P, Kona SSR, Chakravarthi VP, Kumar AVNS, Punyakumari B, Rao VH. 2016. Effect of leptin on in vitro development of ovine preantral ovarian follicles. Theriogenology, 85:224-229. Knight PG, Glister C. 2006. TGF-beta superfamily members and ovarian follicle development. Reproduction, 132:191-206.

Kona SSR, Chakravarthi VP, Kumar AVNS, Srividya D, Padmaja K, Rao VH. 2016. Quantitative expression patterns of GDF9 and BMP15 genes in sheep ovarian follicles grown in vivo or cultured in vitro. Theriogenology, 85:315-322.

Lakshminarayana BNV, Praveen Chakravarthi V, Brahmaiah KV, Rao VH. 2014. Quantification of P450 aromatase gene expression in cultured and in vivo grown ovarian follicles in sheep. Small Rumin Res, 117:66-72.

Langbeen A, Van Ginneken C, Fransen E, Bosmans E, Leroy JLMR, Bols PEJ. 2016. Morphometrical analysis of preantral follicular survival of VEGF-treated bovine ovarian cortex tissue following xenotransplantation in an immune deficient mouse model. Anim Reprod Sci, 168:73-85.

Lima IMT, Celestino JJH, Faustino LR, MagalhãesPadilha DM, Rossetto R, Brito IR, Donato MAM, Lopes CAP, Campello CC, Peixoto CA, Figueiredo JR, Rodrigues APR. 2012. Dynamic Medium Containing Kit Ligand and Follicle-Stimulating Hormone Promotes Follicular Survival, Activation, and Growth during Long-Term in vitro Culture of Caprine Preantral Follicles. Cells Tissues Organs, 195:260-271.

Lins TLBG, Cavalcante AYP, Santos JMS, Menezes VG, Barros VRP, Barberino RS, Bezerra MES, Macedo TJS, Matos MHT. 2017. Rutin can replace the use of three other antioxidants in the culture medium, maintaining the viability of sheep isolated secondary follicles. Theriogenology, 89:263-270.

Lopes TA, Costa JJN, Ribeiro RP, Passos JRS, Soares MAA, Filho JGA, Cunha EV, Van den Hurk R, Pinheiro AA, Silva JRV. 2015. Influence of caprine arthritis encephalitis on expression of ovulation related genes and activation of primordial follicles cultured in presence of phytohemagglutinin, epidermal growth factor or both. Small Rumin Res, 123:278-286.

Luz VB, Araujo VR, Duarte ABG, Celestino JJH, Silva TFP, Magalhaes-Padilha DM, Chaves RN, Brito IR, Almeida AP, Campello CC, Feltrin C, Bertolini M, Santos RR, Figueiredo JR. 2012. Eightcell parthenotes originated from in vitro grown sheep preantral follicles. Reprod Sci, 19:1219-1225.

Luz VB, Araújo VR, Duarte ABG, Silva GM, Chaves RN, Brito IR, Serafim MKB, Campello CC, Feltrin C, Bertolini M, Almeida AP, Santos RR, Figueiredo JR. 2013. Kit ligand and insulin-like growth factor I affect the in vitro development of ovine preantral follicles. Small Rumin Res, 115:99-102.

Magalhães DM, Duarte ABG, Araújo VR, Brito IR, Soares TG, Lima IMT, Lopes CAP, Campello CC, Rodrigues APR, Figueiredo JR. 2011. In vitro production of a caprine embryo from a preantral follicle cultured in media supplemented with growth hormone. Theriogenology, 75:182-188.

Magalhães-Padilha DM, Andrade PM, Sales ET, Araujo VR, Lima IMT, Castro SV, Faustino LR, Lopes CAP, Campello CC, Báo SN, Gastal MO, Gastal EL, Figueiredo JR. 2012a. Effect of sequential medium on in vitro culture of goat ovarian cortical tissue. Anim Reprod Sci, 132:159-68.

Magalhães-Padilha DM, Duarte ABG, Araújo VR, Saraiva MVA, Almeida AP, Rodrigues GQ, Matos MHT, Campello CC, Silva JR, Gastal MO, Gastal EL, Figueiredo JR. 2012b. Steady-state level of insulin-like growth factor-I (IGF-I) receptor mRNA and the effect of IGF-I on the in vitro culture of caprine preantral follicles. Theriogenology, 77:206-13.

Magalhaes-Padilha DM, Fonseca GR, Haag KT, Wischral A, Gastal MO, Jones KL, Geisler-Lee J, Figueiredo JR, Gastal EL. 2012. Long-term in vitro culture of ovarian cortical tissue in goats: Effects of FSH and IGF-I on preantral follicular development and FSH and IGF-I receptor mRNA expression. Cell Tissue Res, 350:503-511.

Malki S, van der Heijden GW, O'Donnell KA, Martin SL, Bortvin A. 2014. A role for retrotransposon LINE-1 in fetal oocyte attrition in mice. Dev Cell, 29:521-533.

Mbemya G, Guerreiro D, Silva L, Vieira L, Sousa G, Alves B, Izaguirry A, Santos F, Telefo P, Pessoa O, Smitz J, Figueiredo J, Rodrigues A. 2017. Justicia insularis improves the in vitro survival and development of ovine preantral follicles enclosed in ovarian tissue. $J$ Pharm Pharmacol, 5:668-680.

McLaughlin M, Telfer EE. 2010. Oocyte development in bovine primordial follicles is promoted by activin and FSH within a two-step serum-free culture system. Reproduction, 139:971-8.

Nandi S, Tripathi SK, Gupta PSP, Mondal S. 2017. Effect of metabolic stressors on survival and growth of in vitro cultured ovine preantral follicles and enclosed oocytes. Theriogenology, 104:80-86.

Paes VM, Vieira LS, Cadenas J, Correi HHV, Sa N, Alves BG, Brandao F, Santos RR, Rodrigues APR, Figueiredo JR. 2018. Goat in vitro follicular response to insulin concentration is affected by base medium and follicular stage. Small Rumin Res, In press; 
doi:10.1016/j.smallrumres.2018.11.001.

Passos MJ, Vasconcelos GL, Silva AWB, Brito IR, Saraiva MVA, Magalhães DM, Costa JJN, Donato MAM, Ribeiro RP, Cunha EV, Peixoto CA, Campello CC, Figueiredo JR, van den Hurk R, Silva JRV. 2013. Accelerated growth of bovine preantral follicles in vitro after stimulation with both $\mathrm{FSH}$ and BMP-15 is accompanied by ultrastructural changes and increased atresia. Theriogenology, 79:1269-1277.

Passos JRS, Costa JJN, Cunha EV, Silva AWB, Ribeiro RP, Souza GBD, Barroso PAA, Dau AMP, Saraiva MVA, Gonçalves PBD, Van den Hurk R, Silva JRV. 2016. Protein and messenger RNA expression of interleukin 1 system members in bovine ovarian follicles and effects of interleukin $1 \beta$ on primordial follicle activation and survival in vitro. Domest Anim Endocrinol, 54:48-59.

Pessoa AFC, Rocha RMP, Brito IR, Silva GM, Chaves RN, Magalhães-Padilha DM, Campello CC, Rodrigues APR, Nunes-Pinheiro DCS, Figueiredo JR. 2014. Effect of morphological integrity, period, and type of culture system on the in vitro development of isolated caprine preantral follicles. Theriogenology, 82:312-317.

Portela AMLR, Ribeiro RP, Costa JJN, Rossi RODS, Passos JRS, Vasconcelos GL, Donato MAM, Peixoto CA, Saraiva MVA, Van den Hurk R., Silva JRV. 2014. Effects of different concentrations of concavalin A and follicle stimulating hormone on goat primordial follicles activation, survival and gene expression. Small Rumin Res, 116:183-191.

Praveen Chakravarthi V, Kona SSR, Siva Kumar AVN, Bhaskar M, Rao VH. 2015. Quantitative expression of antiapoptotic and proapoptotic genes in sheep ovarian follicles grown in vivo or cultured in vitro. Theriogenology 83:590-595.

Rocha RMP, Lima LF, Alves AMCV, Celestino JJH, Matos MHT, Lima-Verde IB, Bernuci MP, Lopes CAP, Báo SN, Campello CC, Rodrigues APR, Figueiredo JR. 2013. Interaction between melatonin and follicle-stimulating hormone promotes in vitro development of caprine preantral follicles. Domest Anim Endocrinol, 44:1-9.

Rocha RM, Alves AMCV, Lima, LF, Duarte ABG, Chaves RN, Brito IR, Costa EC, Bernuci MP, Rosae-Silva AC, Xu M, Rodrigues AP, Campello CC, Figueiredo JR. 2014. Is the mouse follicle culture a good model for the goat with respect to the development of preantral follicles in vitro? Domest Anim Endocrinol, 49:27-30.

Rossetto R, Saraiva M, Santos R, Silva C, Faustino L, Chaves R, Brito I, Rodrigues G, Lima I, Donato M, Peixoto C, Figueiredo J. 2012. Effect of medium composition on the in vitro culture of bovine pre-antral follicles: morphology and viability do not guarantee functionality. Zygote, 21:125-128.

Rossetto R, Saraiva MVA, Bernuci MP, Silva GM, Brito IR, Alves AMCV, Magalhães-padilha DM, Báo SN, Campello CC, Rodrigues APR, Figueiredo JR. 2016. Impact of insulin concentration and mode of FSH addition on the in vitro survival and development of isolated bovine preantral follicles. Theriogenology,
86:1137-1145.

Sadeghnia S, Akhondi MM, Hossein G, Mobini S, Hosseini L, Naderi MM, Boroujeni SB, Sarvari A, Behzadi B, Shirazi A. 2016. Development of sheep primordial follicles encapsulated in alginate or in ovarian tissue in fresh and vitrified samples. Cryobiology, 72:100-105.

Santos RR, Schroevers EJ, Roelen BAJ. 2014. Usefulness of bovine and porcine IVM/IVF models for reproductive toxicology. Reprod Biol Endocrinol, 12:117.

Saraiva MVA, Rossetto R, Brito IR, Celestino JJH, Silva CMG, Faustino LR, Almeida AP, Bruno JB, Magalhaes DM, Matos MHT, Campello CC, Figueiredo JR. 2010. Dynamic medium produces caprine embryo from preantral follicles grown in vitro. Repro. Sci, 17:1135-1143.

Saraiva MVA, Celestino JJH, Araújo VR, Chaves RN, Almeida AP, Lima-Verde IB, Duarte ABG, Silva GM, Martins FS, Bruno JB, Matos MHT, Campello CC, Silva JRV, Figueiredo JR. 2011. Expression of follicle-stimulating hormone receptor (FSHR) in goat ovarian follicles and the impact of sequential culture medium on in vitro development of caprine preantral follicles. Zygote, 19:205-14.

Silva JR, Lucci CM, Carvalho FC, Bao SN, Costa SH, Santos RR, Figueiredo JR. 2000. Effect of coconut water and Braun-Collins solutions at different temperatures and incubation times on the morphology of goat preantral follicles preserved in vitro. Theriogenology, 54:809-822.

Silva CMG, Castro SV, Faustino LR, Rodrigues GQ, Brito IR, Rossetto R, Saraiva MVA, Campello CC, Lobo CH, Souza CEA, Moura AAA, Donato MAM, Peixoto CA, Figueiredo JR. 2013. The effects of epidermal growth factor (EGF) on the in vitro development of isolated goat secondary follicles and the relative mRNA expression of EGF, EGF-R, FSH-R and P450 aromatase in cultured follicles. Res Vet Sci, 94:453-61.

Silva GM, Rossetto R, Chaves RN, Duarte ABG, Araújo VR, Feltrin C, Bernuci MP, Anselmo-Franci JA, Xu M, Woodruff TK, Campello CC, Figueiredo JR. 2014. In vitro development of secondary follicles from pre-pubertal and adult goats cultured in twodimensional or three-dimensional systems. Zygote, 26:1-10.

Silva JR, van den Hurk R, Figueiredo JR. 2016. Ovarian follicle development in vitro and oocyte competence: advances and challenges for farm animals. Domest Anim Endocrinol 55:123-135.

Silva AWB, Ribeiro RP, Menezes VG, Barberino RS, Passos JRS, Dau AMP, Costa JJN, Melo LRF, Bezerra FTG, Donato MAM, Peixoto CA, Matos MHT, Gonçalves PBD, Van den Hurk R, Silva JRV. 2017a. Expression of TNF- $\alpha$ system members in bovine ovarian follicles and the effects of TNF- $\alpha$ or dexamethasone on preantral follicle survival, development and ultrastructure in vitro. Anim Reprod Sci, 182:56-68.

Silva GM, Brito IR, Sales AD, Aguiar FLN, Duarte ABG, Araújo VR, Vieira LA, Magalhães-padilha 
DM, Lima LF, Alves BG, Wheeler MB, Figueiredo JR. 2017b. In vitro growth and maturation of isolated caprine preantral follicles : Influence of insulin and FSH concentration, culture dish, coculture, and oocyte size on meiotic resumption. Theriogenology, 90:32-41.

Smith P, Wilhelm D, Rodgers RJ. 2014. Development of mammalian ovary. J Endocrinol, 221:R145-161.

Sun J, Li X. 2013. Growth and antrum formation of bovine primary follicles in long-term culture in vitro. Reprod Biol. 13:221-228.

Tan C, Bian C, Yang D, Li N, Wu ZF, Hu XX. 2017. Application of genomic selection in farm animal breeding. Yi Chuan, 39:1033-1045.

Tang K, Yang W, Li X, W u CJ, Sang L, Yang L. 2012. GDF-9 and bFGF enhance the effect of FSH on the survival, activation, and growth of cattle primordial follicles. Anim Reprod Sci. 131:129-34.

Thatcher WW. 2017. A 100-year review: historical development of female reproductive physionlogy in dairy cattle. J Dairy Sci, 100:10272-10291.

Wang JJ, Ge W, Liu JC, Klinger FG, Dyce PW, De Felici M, Shen W. 2017. Complete in vitro oogenesis: retrospects and prospects. Cell Death Diff, 24:18451852.

Yamamoto K, Otoi T, Koyama $\mathbf{N}$, Horikita $\mathbf{N}$, Tachikawa S, Miyano T. 1999. Development of live young from bovine small oocytes after growth, maturation and fertilization in vitro. Theriogenology 52, 81-89.

Yoon SJ, Kim KH, Chung HM, Choi DH, Lee WS, Cha KY, Lee KA. 2006. Gene expression profiling of early follicular development in primordial, primary, and secondary follicles. Fertil Steril, 85:193-203.

Zoheir KA, Harisa GI, Allam AA, Yang L, Li X, Liang A, Abd-rabou AA, Harrath AH. 2017. Effect of alpha lipoic acid on in vitro development of bovine secondary preantral follicles. Theriogenology, 88:124130. 